OPEN ACCESS

Edited by:

Fu Wang,

Xi'an Jiaotong University, China

Reviewed by:

Jason Dowling,

Commonwealth Scientific and Industrial Research Organisation

(CSIRO), Australia

Chen Liu,

Army Medical University, China

*Correspondence:

Xinye $\mathrm{Ni}$

nxy@njmu.edu.cn

Jianhua Yang

yangjianhua@nwpu.edu.cn

Specialty section:

This article was submitted to

Cancer Imaging

and Image-directed Interventions,

a section of the journal

Frontiers in Oncology

Received: 08 September 2020

Accepted: 01 February 2021

Published: 12 March 2021

Citation:

Sun H, Fan R, Li C, Lu Z, Xie K, NiX and Yang $J$ (2021) Imaging Study of

Pseudo-CT Synthesized From

Cone-Beam CT Based on

3D CycleGAN in Radiotherapy.

Front. Oncol. 11:603844.

doi: 10.3389/fonc.2021.603844

\section{Imaging Study of Pseudo-CT Synthesized From Cone-Beam CT Based on 3D CycleGAN in Radiotherapy}

\author{
Hongfei Sun ${ }^{1}$, Rongbo Fan ${ }^{1}$, Chunying $\mathrm{Li}^{2,3,4}$, Zhengda $\mathrm{Lu}^{2,3,4}$, Kai Xie ${ }^{2,3,4}$, Xinye $\mathrm{Ni}^{2,3,4 *}$ \\ and Jianhua Yang ${ }^{1 *}$ \\ ${ }^{1}$ School of Automation, Northwestern Polytechnical University, Xi'an, China, ${ }^{2}$ Department of Radiotherapy, Second People's \\ Hospital of Changzhou, Nanjing Medical University, Changzhou, China, ${ }^{3}$ Department of Radiotherapy, The Center of Medical \\ Physics With Naniing Medical University, Changzhou, China, ${ }^{4}$ Department of Radiotherapy, The Key Laboratory of Medical \\ Physics With Changzhou, Changzhou, China
}

Purpose: To propose a synthesis method of pseudo-CT (CT CycleGAN) images based on an improved 3D cycle generative adversarial network (CycleGAN) to solve the limitations of cone-beam CT (CBCT), which cannot be directly applied to the correction of radiotherapy plans.

Methods: The improved U-Net with residual connection and attention gates was used as the generator, and the discriminator was a full convolutional neural network (FCN). The imaging quality of pseudo-CT images is improved by adding a 3D gradient loss function. Fivefold cross-validation was performed to validate our model. Each pseudo CT generated is compared against the real CT image (ground truth $\mathrm{CT}, \mathrm{CT}_{\mathrm{gt}}$ ) of the same patient based on mean absolute error (MAE) and structural similarity index (SSIM). The dice similarity coefficient (DSC) coefficient was used to evaluate the segmentation results of pseudo CT and real CT. 3D CycleGAN performance was compared to 2D CycleGAN based on normalized mutual information (NMI) and peak signal-to-noise ratio (PSNR) metrics between the pseudo-CT and $\mathrm{CT}_{\mathrm{gt}}$ images. The dosimetric accuracy of pseudoCT images was evaluated by gamma analysis.

Results: The MAE metric values between the CT CycleGAN and the real CT in fivefold crossvalidation are $52.03 \pm 4.26 \mathrm{HU}, 50.69 \pm 5.25 \mathrm{HU}, 52.48 \pm 4.42 \mathrm{HU}, 51.27 \pm 4.56 \mathrm{HU}$, and $51.65 \pm 3.97 \mathrm{HU}$, respectively, and the SSIM values are $0.87 \pm 0.02,0.86 \pm 0.03,0.85 \pm$ $0.02,0.85 \pm 0.03$, and $0.87 \pm 0.03$ respectively. The DSC values of the segmentation of bladder, cervix, rectum, and bone between CT CycleGAN and real CT images are $91.58 \pm$ $0.45,88.14 \pm 1.26,87.23 \pm 2.01$, and $92.59 \pm 0.33$, respectively. Compared with $2 \mathrm{D}$ 
CycleGAN, the 3D CycleGAN based pseudo-CT image is closer to the real image, with NMI values of $0.90 \pm 0.01$ and PSNR values of $30.70 \pm 0.78$. The gamma pass rate of the dose distribution between CT CycleGAN and $\mathrm{CT}_{\text {gt }}$ is $97.0 \%(2 \% / 2 \mathrm{~mm})$.

Conclusion: The pseudo-CT images obtained based on the improved 3D CycleGAN have more accurate electronic density and anatomical structure.

Keywords: pseudo computed tomography (CT), CycleGAN, cone-beam computed tomography (CT), radiotherapy, cervical cancer

\section{INTRODUCTION}

Cervical cancer is one of the most common gynecological malignant tumors. According to statistics released in the 2017 annual meeting of the European Society for Medical Oncology (ESMO), the frequency of new cervical cancer cases was fourth highest in female cancers, and its fatality rate was third-highest (1). The main treatment means for cervical cancer are operation and comprehensive chemoradiotherapy (2). With the development of radiotherapy technology, image-guided radiation therapy (IGRT) has been gradually applied to clinical treatment of cervical cancer (3). In comparison with diagnostic $\mathrm{CT}$, cone-beam CT (CBCT), a commonly used image-guided device, has higher spatial resolution, so it can be used for beam position verification of a patient between fractionated treatments (4). Rigid registration based on gray level or bone landmark is carried out through CBCT images scanned before each treatment and $\mathrm{CT}$ images acquired at the simulation stage $\left(\mathrm{CT}_{\text {sim }}\right)$ to formulate a radiotherapy plan. Then, the setup error in $3 \mathrm{D}$ space can be determined to calibrate patient position (5). Given that the tumor target area of cervical cancer is closely related to the surrounding organs at risk (OARs) (such as the bladder and rectum), bladder filling and gastrointestinal peristalsis will directly affect the location of the tumor target area. The size and prescription dose of planning target volume (PTV) should be adjusted before each treatment to minimize the radiation dose of surrounding normal tissues. CBCT and standard multi-slice CT images are grayscale images that are processed and reconstructed by a computer after X-ray passes through different density tissues and organs, and the radiation energy after X-ray attenuation is measured by a flat panel detector. However, their imaging principles are different. CBCT uses $3 \mathrm{D}$ cone beam scanning instead of sector scan of multi-slice CT to obtain $2 \mathrm{D}$ projection data. Then, $\mathrm{CBCT}$ reconstructs the projection data obtained from different angles. Although the use of X-rays is improved, scattered signals are added, in turn causing the soft tissue resolution of CBCT images to decrease and produce more strip or band artifacts. The electron density is inaccurate and difficult to correct. Softtissue visualization also is hindered by tissue/breathing motion artifacts because of long (30-60 s) image acquisition. Therefore, CBCT images need to be modified to meet the requirements of clinical treatment (6).

Many correction methods are used for CBCT artifacts, including hardware-based pre-processing $(7,8)$ and software- based post-processing methods (9-12). Although these methods have been proven to be able to eliminate artifacts and improve image quality they cannot correct the $\mathrm{HU}$ values in СВСТ images, and comprehensively considering the calculation amount and complexity of the algorithm, additional scanning time consumption, and incidental increased radiation dose, and clinical practicality is necessary. After consulting the literature, two main methods based on image post-processing are currently used for the correction of $\mathrm{HU}$ values in CBCT images. The first method is the image registration-based method, mainly including the deformation field registration and histogram matching methods. Chevillard et al. used an elastic deformation registration algorithm to establish the nonlinear mapping relation between $\mathrm{CBCT}$ and $\mathrm{CT}_{\text {sim }}$, and the $\mathrm{CT}$ image after registration not only had anatomical structure information of CBCT but also accurate electron density of $\mathrm{CT}_{\text {sim }}$ (13). Derksen et al. improved the deformation field registration method and constrained the deformation area by adding the OAR contours acquired by the image segmentation method, to improve registration accuracy between $\mathrm{CBCT}$ and $\mathrm{CT}_{\text {sim }}$ images (14). Abe et al. established the greyscale linear relation between СВCT and CT images by the histogram-matching method to correct Hounsfield unit (HU) values in CBCT images, and the experimental results showed that $\mathrm{CBCT}$ images after histogram matching could be applied to therapeutic plan formation of cervical and prostatic cancer (15). Although this type of method can correct $\mathrm{HU}$ information in the CBCT image, it has high accuracy requirements for the image registration algorithm and matching method, and the setting of an objective function is also complicated. The second method is the pseudo-CT synthesis-based method, which mainly includes machine learning and deep learning-based synthesis methods. Yang et al. proposed the alternate random forest method based on an automatic context model to extract multiscale texture features between $\mathrm{CBCT}$ and $\mathrm{CT}$ image pairs to establish nonlinear mapping relations and save the data model. New CBCT images were input into the trained model in the prediction phase to acquire virtual $\mathrm{CT}$ images with $\mathrm{CBCT}$ anatomical structure (16). Wang et al. used the fuzzy C-means clustering algorithm to classify voxel points inside CBCT images and assign CT values to voxel points according to weight information. Finally, they synthesized complete pseudo-CT images and verified the accuracy of $\mathrm{HU}$ values of pseudo-CT images from the aspect of dosimetry (17). Nevertheless, regardless of whether image registration or machine learning-based methods are used to 
synthesize pseudo-CT images, strict alignment of voxel information between $\mathrm{CBCT}$ and $\mathrm{CT}_{\text {sim }}$ images must be guaranteed and restricted by patient differences in bladder filling degree or soft tissue deformation in different periods of the scanning process. Acquiring CBCT and CT image pairs with completely matched anatomical structures is very difficult in practice.

To solve these problems, scholars have proposed the deep learning-based cycle generative adversarial network (CycleGAN) to synthesize pseudo images (18). Different from the traditional GAN network, CycleGAN is a loop network consisting of two GANs with mirror symmetry. The two GANs share two generators and two discriminators. This network is constrained by introducing a cycle consistency loss function to ensure that the model can effectively learn the nonlinear mapping relationship between unpaired image data in two image domains. Liang et al. applied the CycleGAN network to a pseudo image synthesis task between $\mathrm{CBCT}$ and $\mathrm{CT}_{\text {sim }}$ images and verified the accuracy of head and neck pseudo-CT images from the aspects of anatomical structure and dosimetry, respectively (19). Kida et al. proved that pseudo-CT images synthesized based on CycleGAN could be applied to prostate cancer treatment. Compared with the original CBCT, the image quality of the synthesized pseudo-CT image showed a substantial improvement in HU values, spatial uniformity, and artifact suppression. The anatomical structures of the CBCT image were well preserved in the synthesized image (20). However, these models are all applied to 2D CT image synthesis tasks. Spatial and structural information will be lost if a $2 \mathrm{D}$ convolutional kernel is used. Furthermore, the greater the number of $2 \mathrm{D}$ slices input into the network, the longer the model training time. In addition, due to artifacts caused by various factors in the CBCT image, the image quality will be degraded. Directly using the CycleGAN method to establish the mapping relationship between CBCT and CT images would result in falsely synthesized pseudo-CT images. Therefore, a 3D CycleGAN network carrying residual connection and attention gates was proposed in this study, and the gradient loss function was added into the objective function to further improve the accuracy of the synthesized CT images. The purpose of this study is to prove that electron density and organ relative position of the pseudo-CT images obtained based on improved CycleGAN are more accurate than those obtained by other deep learning methods. The accuracy of pseudo-CT images is also verified in terms of anatomy and dosimetry. The pseudo-CT images obtained by the new method are proved to have more accurate electron density for dose calculation. In this study, we also compared the pseudo-CT images obtained by 2D CycleGAN and 3D CycleGAN to prove the advantages of 3D network in the task of synthesizing pseudoCT images. To avoid GPU memory restrictions imposed on 3D neural networks in training, the network was trained using a 3D image block-based network computing model, which could acquire abundant feature information while improving computing efficiency. On the basis of the literature review, this study is the first to use the 3D CycleGAN method to synthesize the $3 \mathrm{D}$ pseudo-CT from the CBCT image of the pelvic region.

\section{MATERIALS AND METHODS}

\section{Data Acquisition and Image Processing}

A total of 120 sets of CT-CBCT image pairs used for training and prediction were obtained from 120 different patients. All image data selected in this experiment were $3 \mathrm{D}$ volume data of cervical cancer patients undergoing Volumetric Modulated Arc Therapy (VMAT). Among them, 100 cases were used for fivefold crossvalidation to train the model, and the other 20 cases were used for testing. The CT images used in the entire process were obtained in the simulation stage, and the CBCT images were obtained by the patients after one week of treatment. CT images were acquired via an Optima CT520 device produced by GE Corporation (United States). Scanning conditions were as follows: tube voltage $120 \mathrm{kV}$, tube current $220 \mathrm{~mA}$, image size $512 \times 512 \times(102-119)$, and voxel spacing $0.9765 \times 0.9765 \times 3 \mathrm{~mm}^{3}$. CBCT was equipped for an infinity linear accelerator, which was produced by Elekta Corporation (Sweden), was used to scan patients who had already accepted treatment for one week. Scanning conditions were as follows: tube voltage $120 \mathrm{kV}$, tube current $20 \mathrm{~mA}$, image size $410 \times 410 \times(50-76)$, and voxel spacing $1 \times 1 \times 5 \mathrm{~mm}^{3}$. CycleGAN input did not need two-group data with one-to-one registered voxel information. However, to improve the efficiency of operation, facilitate the training of the model, and reduce the interference of background voxel points outside the imaging area on image synthesis, we performed rigid registration based on bone landmarks on CT-CBCT image pairs to be input into the network. Before the model training, we also resampled the $\mathrm{CT}$ and $\mathrm{CBCT}$ images and preprocessed them with bicubic interpolation. Voxel spacing of preprocessed image data was unified as $1 \times 1 \times 1 \mathrm{~mm}^{3}$ and image size as $384 \times 192 \times 192$. The minimum HU value of image data was unified as $-1,000$. For the convenience of GPU memory and acquiring refined image features, a complete $3 \mathrm{D}$ image was divided into $32 \times 32 \times 32$ small image blocks in the experiment as input dimensions of the network model. During the acquisition of the image blocks, step size was set as 16, and an overlapping area occurred between adjacent image blocks, thereby ensuring that all imaging content was distributed in the image blocks and loss of image information was avoided.

\section{Pseudo-Computed Tomography Image Synthesis Based on 3D CycleGAN}

The traditional GAN is unidirectional. CycleGAN used in this experiment was a loop network consisting of one unidirectional $\mathrm{CBCT} \rightarrow \mathrm{CT}$ GAN and another unidirectional $\mathrm{CT} \rightarrow \mathrm{CBCT}$ GAN . The CycleGAN contained two discriminators, in which discriminators 1 and 2 were used to judge the authenticity of the CBCT and CT images, respectively. CycleGAN also included two generators that were each used to generate pseudo-CT and pseudo-CBCT images. By acquiring an input image from original domain $\mathrm{A}$, this model transmitted the input image to the first generator in the form of voxel block, converted it into an image block in target domain $\mathrm{B}$, and reconstructed a complete image. The generated image was also used as the input in the form of 
voxel block to be transmitted into the second generator, converted into an image block in original domain $\mathrm{A}$, and reconstructed into an output image. This output image must be approximate to the original input image in gray level and anatomical structure. Here, the nonlinear mapping relationship between two unpaired image data is set. CBCT and CT images served as input images of the original domain to train two independent GAN networks. An association was established through the cycle consistency loss function to constitute a complete CycleGAN. Its overall network structure is shown in Figure 1.

\section{Generators and Discriminators of 3D CycleGAN}

A CNN with a residual connection has already been proven to have excellent application effects in many image processing tasks (21-23). This CNN expresses network output as a linear superposition of nonlinear transformation of inputs through identity shortcut connection. In comparison with a directly connected convolutional neural network, ResNet directly transmits feature information of the input network along the shortcut, which can protect information integrity to a certain degree, simplifying and clarifying the model learning goal and solving the gradient missing problem in training (24). In addition, the attention gate has been proven to be able to complete the $\mathrm{CBCT} \rightarrow \mathrm{CT}$ image synthesis task well. The model with attention gates uses attention coefficients to highlight image regions with salient features and suppress the feature responses of irrelevant regions during training, that is, effectively suppress the artifact regions in $\mathrm{CBCT}$ images. Interested readers can refer to the paper by Liu et al. (25) on the detailed design of the network with an attention gate. The generator used in this study was a deep convolutional neural network similar to U-Net with residual connection and attention gates, in which $32 \times 32 \times 32$ patch voxel blocks in $\mathrm{CT}_{\text {sim }}$ and $\mathrm{CBCT}$ image domains were used as inputs of the synthesis direction of the two pseudo images in the network. Before each skip connection, the network with attention gates added a gating signal to the output of the encoder and corresponding decoder under each resolution. These signals were used to define the importance of image features at different positions in 3D image space and readjust the output features of the network layer. The patch blocks input into the generative network first passed through three ConvBlock blocks. ConvBlock consisted of two convolutional layers with a step size of 1 and one convolutional layer with a step size of 2 , in which each convolutional layer included conv, BN, and LReLU operations, and the padding was SAME. The output abstract features passed through another three-group concatenate and deconvolutional layers, and the inputs of each concatenate group were the output of the previous convolutional layer and the output after it passed through the attention gate module together with its corresponding ConvBlock. Each deconvolutional layer included deconv, BN, and LReLU operations. Step size was 2 and the padding was SAME. Abstract features passed through the remaining ResNet and convolutional layers of the generator, aiming to enhance network nonlinearity. The dimensions of the convolution kernel used in all network layers were $3 \times 3 \times 3$. The concrete network structure of the generator is shown in Figure 2.

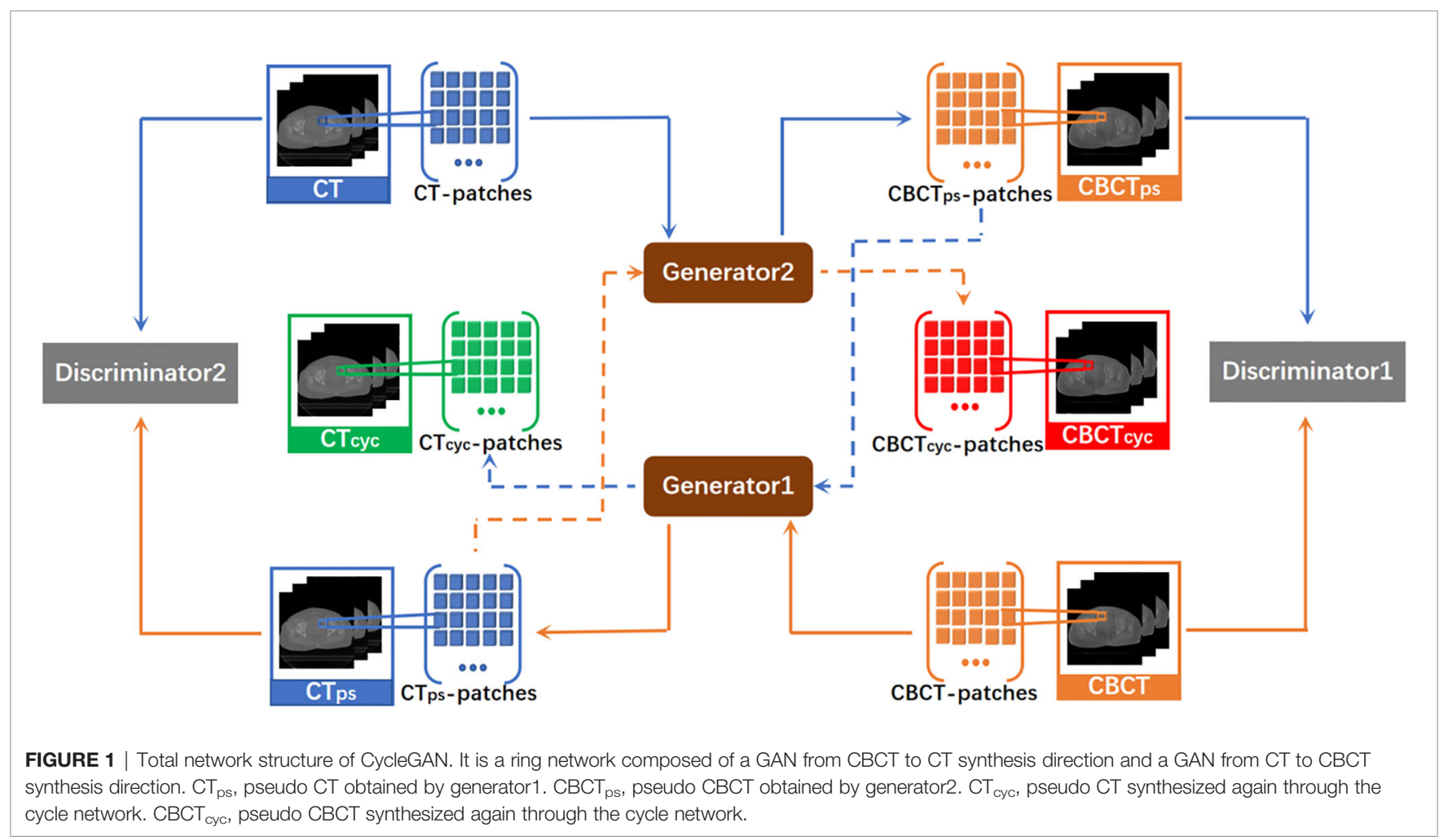


Both discriminators of CycleGAN were conventional full convolutional neural networks $(\mathrm{FCN})$, which received patch blocks in the CBCT and CT image domains as inputs, respectively. Each discriminator contained four convolution layers and three fully connected layers. Each convolutional layer included convolution, BN, and LReLU operations. Dimensions of convolution kernel were $4 \times 4 \times 4$; step sizes were $2,2,2$, and 1 , respectively; and padding was SAME. LReLU served as the activation function in the first two fully connected layers, and the Sigmoid activation function was used in the third fully connected layer to acquire judgment results of the discriminator regarding the authenticity of the input image. The result value was a probability. Feature maps at all layers of the discriminator were 16, 32, 64, 128, 256,128 , and 1 , respectively. The concrete network structure of the discriminator is shown in Figure 3.

\section{Loss Functions of 3D CycleGAN}

The loss function of the CycleGAN network contains two parts: generator loss and discriminator loss functions. The main task of the discriminator is to distinguish real image data from pseudo image data synthesized via a generator. According to the GAN network structure of mirror symmetry, CycleGAN has discriminators in two image domains, where discriminator 1 is used to judge the authenticity of CBCT data. To calculate the loss function, discriminator 1 includes two inputs and two corresponding outputs. Discriminator 1 uses pseudo-CBCT image $\mathrm{G}_{2}\left(\mathrm{X}_{\mathrm{CT}}\right)$ generated by generator 2 as an input to obtain output $\mathrm{D}\left(\mathrm{G}_{2}\left(\mathrm{X}_{\mathrm{CT}}\right)\right)$ and real CBCT image $\mathrm{Y}_{\mathrm{CBCT}}$ as another input to obtain output $\mathrm{D}\left(\mathrm{Y}_{\mathrm{CBCT}}\right)$. Hence, the loss function of discriminator 1 is defined as follows:

$$
L_{D_{1}}=L_{B C E}\left(1, D\left(Y_{C B C T}\right)\right)+L_{B C E}\left(0, D\left(G\left(X_{C T}\right)\right)\right)
$$

where $\mathrm{L}_{\mathrm{BCE}}$ is the binary cross-entropy loss function, which is defined in Formula 2. $\mathrm{Z}$ represents an input image data label, the value of which is taken as 1 or 0 based on data authenticity. $Z$ denotes the probability for the discriminator to predict the input image as a real or pseudo image, and its value range is $[0,1]$.

$$
L_{B C E}\left(Z, Z^{\prime}\right)=-\sum_{i=1}^{N} Z_{i} \log \left(Z_{i}^{\prime}\right)+\left(1-Z_{i}\right) \log \left(1-Z_{i}^{\prime}\right)
$$

Discriminator 2 is used to judge the authenticity of CT data. It also includes two inputs and two outputs, and its loss function is defined as follows:

$$
L_{D_{2}}=L_{B C E}\left(1, D\left(Y_{C T}\right)\right)+L_{B C E}\left(0, D\left(G\left(X_{C B C T}\right)\right)\right)
$$

where $\mathrm{Y}_{\mathrm{CT}}$ is a real CT image, and the corresponding output is $\mathrm{D}$ $\left(\mathrm{Y}_{\mathrm{CT}}\right) . \mathrm{G}\left(\mathrm{X}_{\mathrm{CBCT}}\right)$ is a pseudo image generated by generator 1 . The corresponding output is $\mathrm{D}\left(\mathrm{G}\left(\mathrm{X}_{\mathrm{CBCT}}\right)\right)$, and the total loss function of this discriminator is shown in Formula 4.

$$
L_{D}=L_{D} 1+L_{D_{2}}
$$

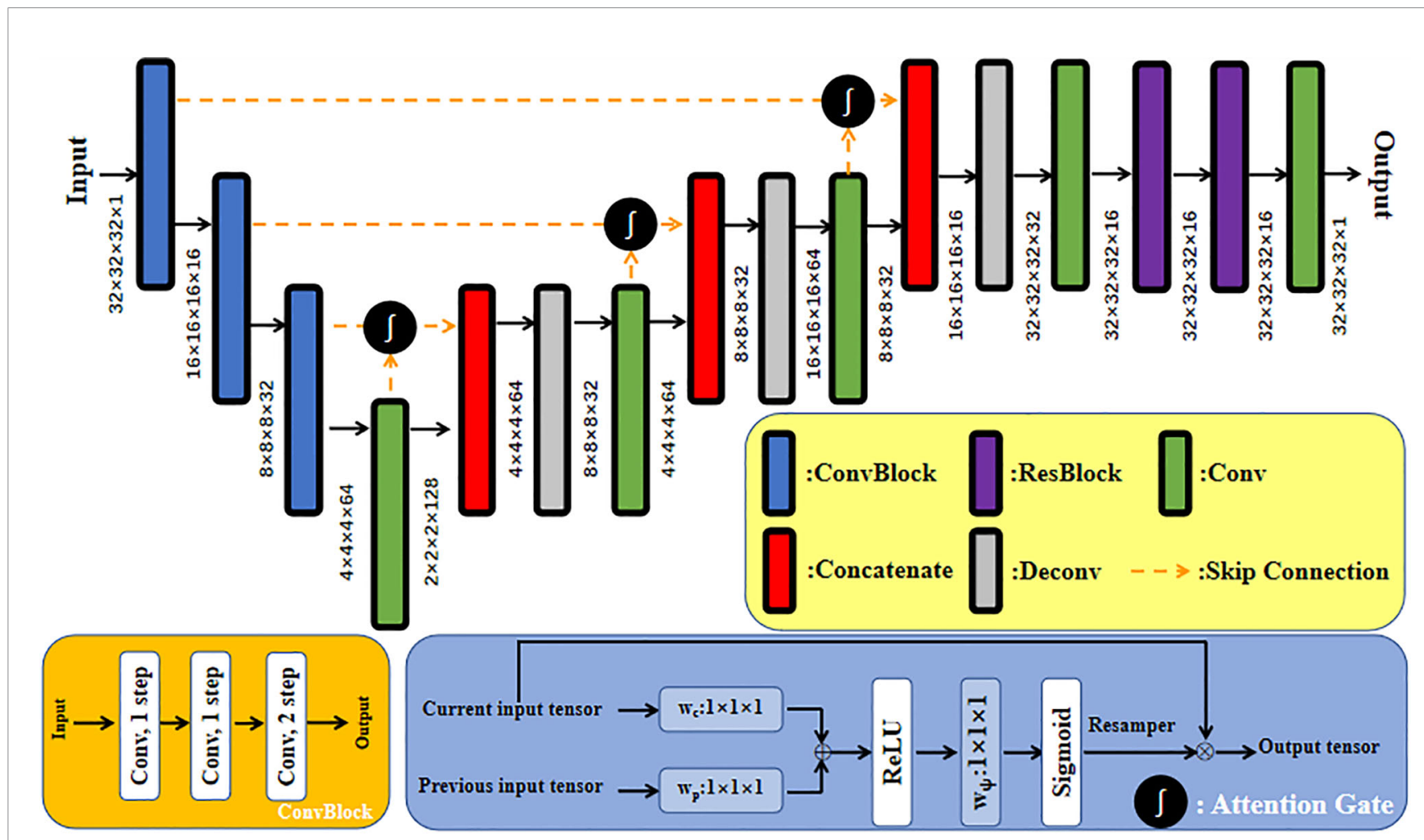

FIGURE 2 | U-Net network structure of generator with residual connection and attention gates. 


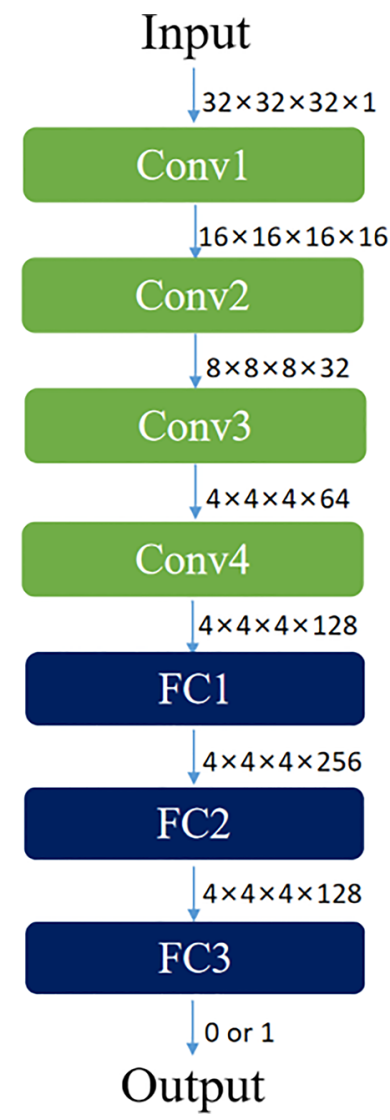

FIGURE 3 | Network structure diagram of discriminator based on FCN.

The main task of the generator is to acquire pseudo image data which is as approximate as possible to real input image data in the aspects of gray level and anatomical structure, to perplex the generator. The loss function of the generator includes adversarial, cycle consistency, and gradient losses. For generator 1, its adversarial loss is the binary cross-entropy $\mathrm{L}_{\mathrm{BCE}}\left(1, \mathrm{D}\left(\mathrm{G}_{1}\left(\mathrm{X}_{\mathrm{CBCT}}\right)\right)\right)$ of the probability for discriminator 2 to discriminate pseudo-CT image $\mathrm{G}_{1}\left(\mathrm{X}_{\mathrm{CBCT}}\right)$ generated by generator 1 as a real image. The probability is 1 . Similarly, the loss function of generator 2 is binary cross-entropy $\mathrm{L}_{\mathrm{BCE}}\left(1, \mathrm{D}\left(\mathrm{G}_{2}\right.\right.$ $\left.\left(\mathrm{X}_{\mathrm{CT}}\right)\right)$ ) of probability $\mathrm{D}\left(\mathrm{G}_{2}\left(\mathrm{X}_{\mathrm{CT}}\right)\right)$ and 1 .

In addition to the adversarial loss of classical GAN, the CycleGAN network also has cycle loss (26). The network needs to ensure that the generated image reserves the characteristics of the original image. Thus, if one generator in the network is used to generate a $3 \mathrm{D}$ pseudo image, then the other generator should be used to recover the original input $3 \mathrm{D}$ image data as much as possible. This process needs to satisfy cycle consistency. L1 loss was used in this study as cycle consistency loss. Cycle consistency losses of generators 1 and 2 are defined as follows:

$$
L_{c y c-G_{1}}=\sum_{i=1}^{N}\left\|G_{2}\left(G_{1}\left(X_{C B C T}\right)\right)-X_{C B C T}\right\|_{2}
$$

$$
L_{c y c-G_{2}}=\sum_{i=1}^{N}\left\|G_{1}\left(G_{2}\left(X_{C T}\right)\right)-X_{C T}\right\|_{2}
$$

In addition, the L1 loss function used in the cycle consistency loss will lead to image fuzziness, a gradient loss function was added in this study to enhance 3D gradient similarity between pseudo image data synthesized by the generator and real image data so that the texture information of the pseudo image can be as accurate as possible. Gradient loss functions $\mathrm{L}_{\mathrm{GL}-\mathrm{CT}}$ and $\mathrm{L}_{\mathrm{GL}-\mathrm{CBCT}}$ are defined in Formulas (7) and (8), respectively.

$$
\begin{aligned}
L_{G L-C T}= & \left.|| \nabla G_{1}\left(X_{C B C T}\right)_{x}|-| \nabla X_{C T} x\right|^{2}+\| \\
& \nabla G_{1}\left(X_{C B C T}\right)_{y}|-| \nabla X_{C T} y||^{2}+|| \\
& \nabla G_{1}\left(X_{C B C T}\right)_{z}|-| \nabla X_{C T} z||^{2} \\
L_{G L-C B C T}= & \| \nabla G_{2}\left(X_{C T}\right)_{x}|-| \nabla X_{C B C T} x||^{2}+|| \\
& \nabla G_{2}\left(X_{C T}\right)_{y}|-| \nabla X_{C B C T} y||^{2}+|| \\
& \nabla G_{2}\left(X_{C T}\right)_{z}|-| \nabla X_{C B C T} z||^{2}
\end{aligned}
$$

In summary, the total loss function of the generator is as follows:

$$
\begin{aligned}
L_{G}= & L_{B C E}\left(1, D\left(G_{1}\left(X_{C B C T}\right)\right)\right)+L_{B C E}\left(1, D\left(G_{2}\left(X_{C T}\right)\right)\right) \\
& +\lambda_{1} L_{C y c-G_{1}}+\lambda_{2} L_{C y c-G_{2}}+\lambda_{3} L_{G L-C T}+\lambda_{4} L_{G L-C B C T}
\end{aligned}
$$

where $\lambda_{1}=\lambda_{2}=10$ and $\lambda_{3}=\lambda_{4}=0.5$.

\section{Cross-Validation of the Trained Model}

To validate the model's performance, a fivefold cross-validation technique was used for training and testing steps, where 100 cases are randomly partitioned into five groups. For each experiment, four groups (including 80 cases) are selected for testing the trained model. Once the model is trained, it is applied to each test subject's CBCT image to generate the pseudo CT. Pseudo CT synthesized based on a 3D GAN with a U-Net generator $\left(\mathrm{CT}_{\text {unet-GAN }}\right)$ and $3 \mathrm{D}$ GAN with an FCN generator $\left(\mathrm{CT}_{\mathrm{FCN}-\mathrm{GAN}}\right)$ were selected as the control experiments to verify the accuracy of the pseudo-CT images acquired based on the improved CycleGAN $(27,28)$.

The accuracy of each subject's pseudo CT and real CT was evaluated using the voxel-wise mean absolute error (MAE) calculated in the pelvic region:

$$
\operatorname{MAE}\left(C T_{\text {real }}, C T_{p s}\right)=\frac{1}{N} \sum_{i=1}^{N}\left|C T_{\text {real }}(i)-C T_{p s}(i)\right|
$$

where the $\mathrm{N}$ is the total number of the voxels in the pelvic region of the CT. The $\mathrm{CT}_{\text {real }}$ is the real image scanned by a CT machine. The $\mathrm{CT}_{\mathrm{ps}}$ is the pseudo CT obtained based on the improved CycleGAN. Another metric used to evaluate the prediction accuracy of the model is the structural similarity coefficient (SSIM). Its mathematical definition is as follows: 


$$
S S I M=\frac{\left(2 \mu_{r} \mu_{p}+C_{1}\right)\left(2 \delta_{r p}+C_{2}\right)}{\left(\mu_{r}^{2}+\mu_{p}^{2}+C_{1}\right)\left(\delta_{r}^{2}+\delta_{p}^{2}+C_{2}\right)}
$$

$\mu_{r}$ and $\mu_{p}$ are the mean values of HU of real CT image and pseudo-CT image, respectively, $\delta_{r}$ and $\delta_{p}$ is the variance of $\mathrm{HU}$ values of real CT image and pseudo-CT image, respectively, $\delta_{r p}$ is the covariance, the parameters $C_{1}=\left(k_{1} L\right)^{2}$ and $C_{2}=\left(k_{2} L\right)^{2}$ are two variables to stabilize the division with weak denominators, $\mathrm{L}$ is the range of $\mathrm{HU}$ values in CT image. $\mathrm{k}_{1}=0.01, \mathrm{k}_{2}=0.02$. The SSIM value range is $[0,1]$, the closer the value is to 1 , the greater the similarity between the two images.

\section{Evaluation}

Dice similarity coefficient (DSC) (29) was used to evaluate the accuracy difference between pseudo-CT images obtained by different methods and $\mathrm{CT}_{\mathrm{gt}}$ images on multiple organs at risk. In this study, the distinct curve-guided FCN proposed by $\mathrm{He}$ et al. was used to segment the OARs in the pelvic region of the pseudo-CT images and CBCT images (30). Segmentation accuracies of bladder and uterus regions in the pseudo-CT images were evaluated through DSC. The ground truth is the contour of the bladder, uterus, rectum, and bone regions manually segmented on the $\mathrm{CT}_{\mathrm{gt}}$ images. The overlapping ratio of OARs between pseudoCT images obtained through different algorithms and $\mathrm{CT}_{\mathrm{gt}}$ images was calculated. An accurate segmentation result should have a high overlapping ratio of organ volumes. DSC is defined as follows:

$$
D S C=\frac{2\left|L_{C T_{g t}} \cap L_{C T_{p s}}\right|}{\left|L_{C T_{g t}}\right|+\left|L_{C T_{p s}}\right|}
$$

where $L_{C T_{g t}}$ and $L_{C T_{p s}}$ represent segmentation results of OARs in real CT and pseudo-CT images acquired through different algorithms, respectively. The closer the DSC value to 1 , the higher the similarity between OAR regions in pseudo-CT image and the corresponding region in $\mathrm{CT}_{\mathrm{gt}}$ image.

Two quantitative measurement methods, namely, normalized mutual information (NMI) (31), peak signal-to-noise ratio (PSNR) (32), and were used in this study to evaluate the accuracy of pseudo-CT images obtained through $3 \mathrm{D}$ and $2 \mathrm{D}$ CycleGAN in anatomical structure.

The first quantitative index is NMI, which is used to evaluate the similarity between pseudo-CT images acquired through different methods and $\mathrm{CT}_{\mathrm{gt}}$. Its expression is as follows:

$$
\begin{gathered}
\operatorname{NMI}\left(C T_{g t}, C T_{p s}\right)=\frac{2 I\left(C T_{g t}, C T_{p s}\right)}{H\left(C T_{g t}\right)+H\left(C T_{p s}\right)} \\
I\left(C T_{g t}, C T_{p s}\right)= \\
H\left(C T_{g t}\right) \\
-H\left(C T_{g t} \mid C T_{p s}\right)=H\left(C T_{g t}\right)-H\left(C T_{p s} \mid C T_{g t}\right)
\end{gathered}
$$

$\mathrm{I}\left(\mathrm{CT}_{\mathrm{gt}}, \mathrm{CT}_{\mathrm{ps}}\right)$ is the mutual information value between pseudo-CT and ground truth $\mathrm{CT}$ images. $\mathrm{H}\left(\mathrm{CT}_{\mathrm{gt}}\right)$ and $\mathrm{H}$ $\left(\mathrm{CT}_{\mathrm{ps}}\right)$ are information entropies. The closer the NMI value is to 1 , the better the image registration effect.
The second quantitative index is PSNR, the formula of which is as follows:

$P S N R=20 \log _{10}\left(\frac{M A X_{I}}{\sqrt{\frac{1}{X Y Z} \sum_{x=0}^{X-1} \sum_{y=0}^{Y-1} \sum_{z-1}^{Z-1}\left|I_{g t}(x, y, z)-I_{p s}(x, y, z)\right|^{2}}}\right)$

In Formula (13), $I_{g t}$ and $I_{p s}$ denote $\mathrm{CT}_{\mathrm{gt}}$ and pseudo-CT images, respectively. $\mathrm{X}, \mathrm{Y}$, and $\mathrm{Z}$ represent image sizes. $M A X_{I}$ is the maximum gray value in the CT image. The greater the PSNR value, the more approximate the synthesized pseudo-CT image to the $\mathrm{CT}_{\mathrm{gt}}$ image.

Pseudo-CT images synthesized through different deep learning methods and CT images acquired through registration were imported into the Monaco planning system (Elekta, Sweden), where the latter was selected as the ground truth image for dosimetry verification. Three radiotherapists with rich clinical experience jointly re-delineated PTVs and OARs on a $\mathrm{CT}_{\mathrm{gt}}$ image and copied them onto pseudo-CT and CBCT images. VMAT radiotherapy plans were prepared respectively on $\mathrm{CT}_{\mathrm{gt}}$ and pseudo-CT images acquired through three deep learning methods based on Monte Carlo algorithm. The dose of the original $4500 \mathrm{cGy} / 25 \mathrm{~F}$ prescription was modified into the new prescription dose $3600 \mathrm{cGy} / 20 \mathrm{~F}$. Dose calculation was implemented via the Monte Carlo algorithm based on the $\mathrm{CT}_{\mathrm{gt}}$ image, and then the optimized plan was copied onto different pseudo-CT images and CBCT image after conforming to clinical requirements. To compare the difference between pseudo-CT and $\mathrm{CT}_{\mathrm{gt}}$ images in the radiotherapy plan, the $3600 \mathrm{cGy}$ prescription dose with 95\% PTV was used as the passing criterion of the plan. The doses in PTV and OARs of cervical cancer patients, which were obtained based on pseudo-CT and $\mathrm{CT}_{\mathrm{gt}}$ images under the same optimization conditions of VMAT treatment in the planning system, were compared. OARs included bladder, femoral head, and small intestine. The main dosimetry evaluation indexes included dose-volume histogram (DVH), dose covering 98\% of the PTV (D98\%), mean dose (Dmean), and dose to $2 \%$ of the PTV (D2\%). In addition, based on the dose distribution of $\mathrm{CT}_{\mathrm{gt}}$, the pass rate of $\gamma$ analysis was evaluated for the central level dose of pseudo CT obtained by three methods (33). The parameter standard was $2 \% / 2 \mathrm{~mm}$ (dose difference $2 \%$, distance difference $2 \mathrm{~mm}$ ).

\section{RESULTS}

\section{Evaluation of Anatomical Structure}

As for anatomical structure verification, Table $\mathbf{1}$ provides a summary of MAE and SSIM metrics computed based on the real and different pseudo $\mathrm{CT}$ for each fold in the fivefold validation. Compared with other GAN methods, the pseudoCT synthesized by the improved CycleGAN method proposed in this study has higher accuracy, and its MAE value decreases and SSIM value increases. This finding indicates that the results obtained by CycleGAN with gradient information in the 
TABLE 1 | Metric results of MAE and SSIM computed between real CT image and different pseudo-CT images for fivefold cross-validation.

\begin{tabular}{|c|c|c|c|c|c|c|}
\hline & Metric & Fold 1 & Fold 2 & Fold 3 & Fold 4 & Fold 5 \\
\hline \multirow[t]{2}{*}{ CycleGAN } & MAE & $52.03 \pm 4.26 \mathrm{HU}$ & $50.69 \pm 5.25 \mathrm{HU}$ & $52.48 \pm 4.42 \mathrm{HU}$ & $51.27 \pm 4.56 \mathrm{HU}$ & $51.65 \pm 3.97 \mathrm{HU}$ \\
\hline & SSIM & $0.87 \pm 0.02$ & $0.86 \pm 0.03$ & $0.85 \pm 0.02$ & $0.85 \pm 0.03$ & $0.87 \pm 0.03$ \\
\hline \multirow[t]{2}{*}{ Unet-GAN } & MAE & $55.36 \pm 5.42 \mathrm{HU}$ & $56.33 \pm 5.02 \mathrm{HU}$ & $57.18 \pm 5.23 \mathrm{HU}$ & $57.22 \pm 5.06 \mathrm{HU}$ & $56.54 \pm 5.58 \mathrm{HU}$ \\
\hline & SSIM & $0.80 \pm 0.03$ & $0.82 \pm 0.03$ & $0.82 \pm 0.02$ & $0.81 \pm 0.02$ & $0.81 \pm 0.02$ \\
\hline \multirow[t]{2}{*}{ FCN-GAN } & MAE & $67.63 \pm 5.49 \mathrm{HU}$ & $65.36 \pm 6.02 \mathrm{HU}$ & $62.34 \pm 6.35 \mathrm{HU}$ & $62.97 \pm 7.19 \mathrm{HU}$ & $63.64 \pm 7.87 \mathrm{HU}$ \\
\hline & SSIM & $0.75 \pm 0.04$ & $0.74 \pm 0.03$ & $0.78 \pm 0.02$ & $0.79 \pm 0.02$ & $0.77 \pm 0.03$ \\
\hline
\end{tabular}

unpaired CBCT and CT image synthesis tasks are closer to the real CT images with higher quality.

The graphical results of $\mathrm{CT}_{\mathrm{gt}}$ image and pseudo-CT images acquired through different deep learning methods in three axial directions are presented in Figure 4. Here, $\mathrm{CT}_{\mathrm{gt}}$ was a CT image after registration of $\mathrm{CBCT}$ and $\mathrm{CT}_{\text {sim }}$ images. The specific registration method has been clarified in the II. D.section. Given that the 3D FCN-GAN method could largely acquire pseudo-CT images $\left(\mathrm{CT}_{\mathrm{FCN}-\mathrm{GAN}}\right)$, but its imaging quality was poor, the resolution of soft tissues was low and the bone region underwent deformations to different degrees. In comparison with the former, a pseudo-CT image $\left(\mathrm{CT}_{\text {unet-GAN }}\right)$ acquired based on the 3D Unet-GAN method had a better effect, but the skeleton region still experienced partial deformation and some soft tissues were inaccurate. The pseudo-CT image $\left(\mathrm{CT}_{\text {CycleGAN }}\right)$ acquired based on the $3 \mathrm{D}$ CycleGAN method was the most approximate to $\mathrm{CT}_{\mathrm{gt}}$ image in anatomical structure, and textures of soft tissues and organs in this image were similar to those in the $\mathrm{CT}_{\mathrm{gt}}$ image. Figure $\mathbf{5}$ shows $\mathrm{CT}$ value difference plots between pseudo-CT images acquired through different deep learning methods and $\mathrm{CT}_{\mathrm{gt}}$, where 5(a) shows the $\mathrm{CT}$ value difference plot between $\mathrm{CT}_{\mathrm{gt}}$ and $\mathrm{CT}_{\text {CycleGAN. Their } \mathrm{CT}}$ value difference in the soft tissue region was within $50 \mathrm{HU}$. Figure 5B displays the CT value difference plot between $\mathrm{CT}_{\mathrm{gt}}$ and $\mathrm{CT}_{\text {unet-GAN }}$, and $5(\mathrm{c})$ is that between $\mathrm{CT}_{\mathrm{gt}}$ and $\mathrm{CT}_{\mathrm{FCN}-\mathrm{GAN}}$ $\mathrm{CT}$ values of the latter two pseudo-CT images were different from those of $\mathrm{CT}_{\mathrm{gt}}$ in the skeleton and soft tissue regions to different degrees. Table 2 presents the DSC measurement results of $3 \mathrm{D}$ volume overlapping differences of ladder, uterus, rectum, and bone regions between real CT and different pseudo-CT images of predicted volume data of 20 cases. Different OARs in $\mathrm{CT}_{\text {CycleGAN }}$ and $\mathrm{CT}_{\mathrm{gt}}$ images had higher DSC values.

Figure 6 shows the comparison result of pseudo CT obtained based on 3D and 2D CycleGAN. The pseudo-CT images
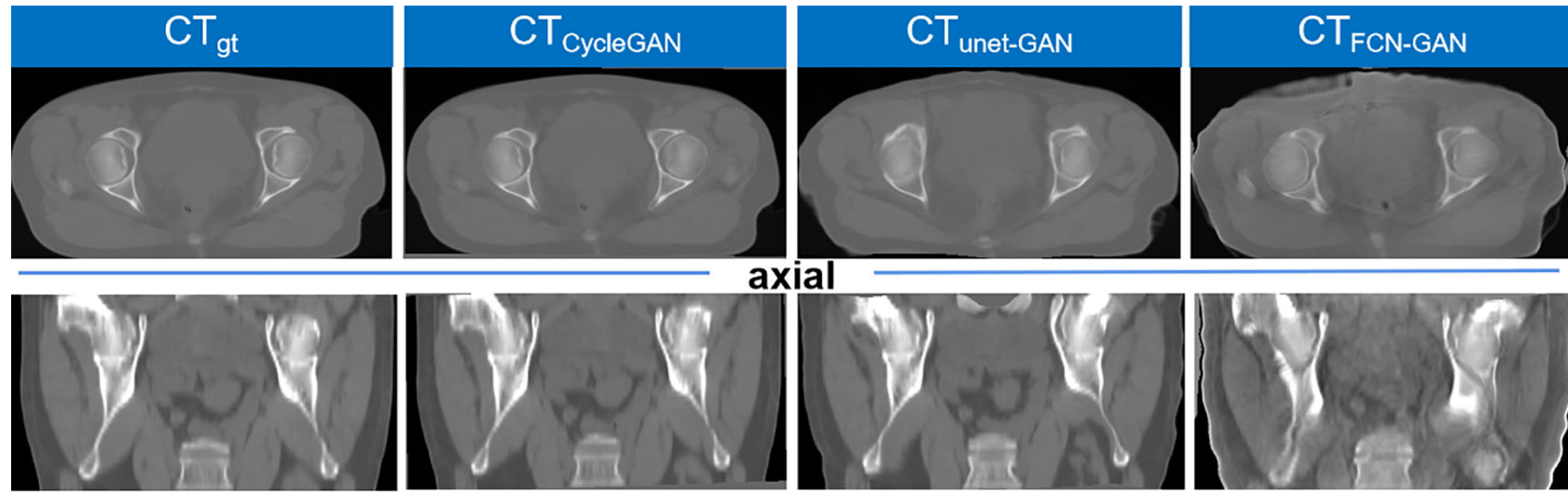

axial
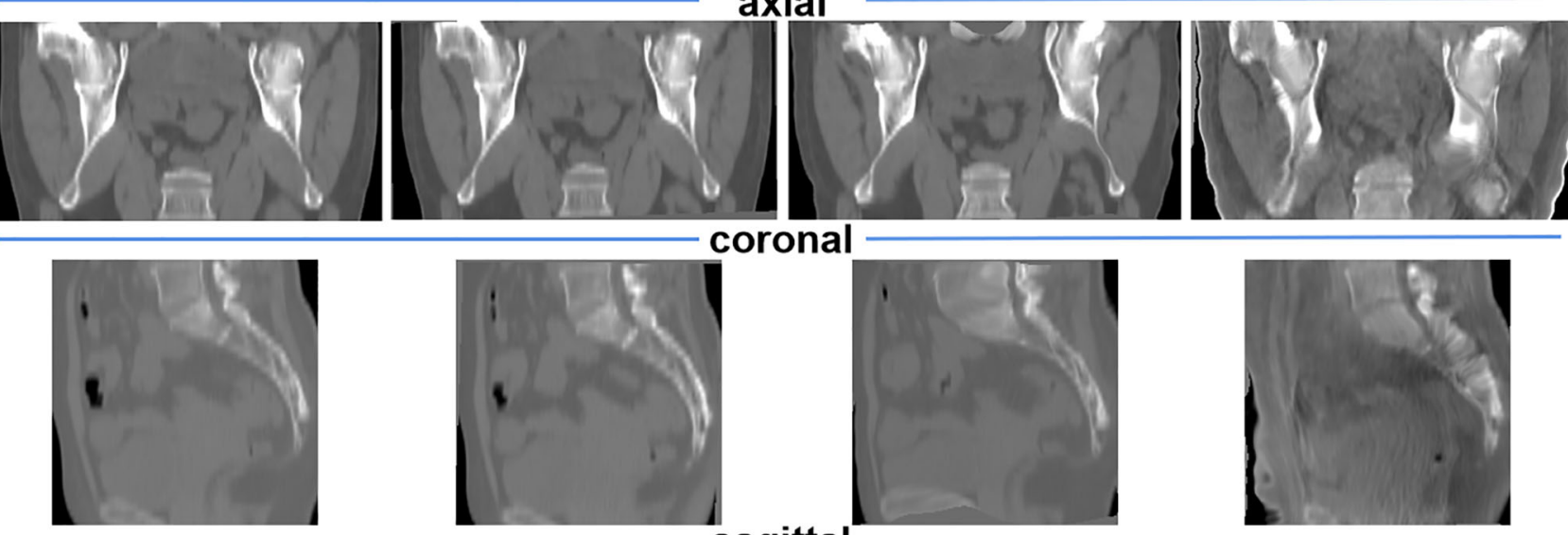

coronal

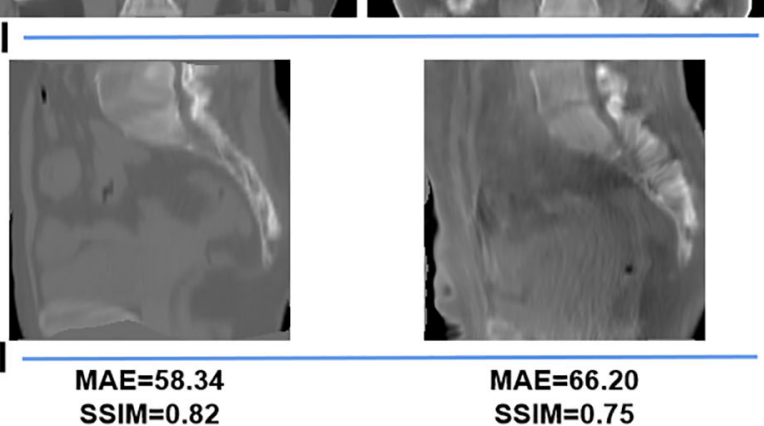

FIGURE 4 | Comparison of the results of $\mathrm{CT}_{\mathrm{gt}}$ images and pseudo-CT images obtained based on different deep learning methods in the axial, coronal, and sagittal directions. $\mathrm{CT}_{\mathrm{gt}}$ : The real CT images. CT CycleGAN: Pseudo CT obtained based on CycleGAN. CT unet-GAN: Pseudo CT obtained by GAN with U-net generator. $\mathrm{CT}_{\text {FCN-GAN: }}$ Pseudo CT obtained by GAN with FCN generator. MAE: mean absolute error. SSIM: structural similarity coefficient. 


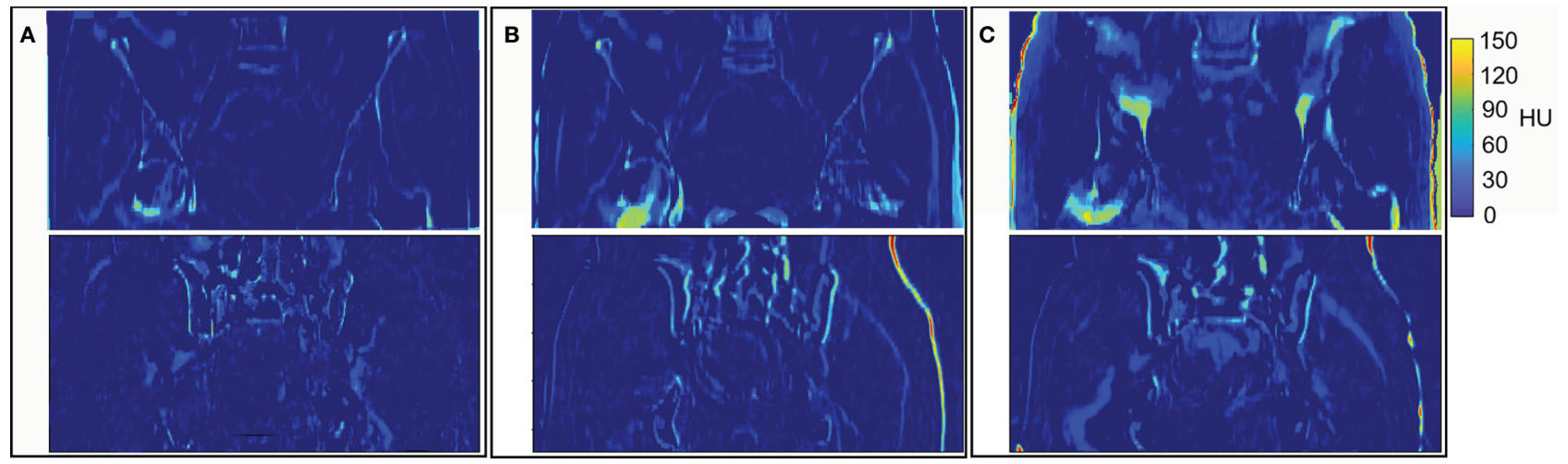

FIGURE 5 | Results of HU values difference between $\mathrm{CT}_{\mathrm{gt}}$ and pseudo $\mathrm{CT}$ obtained by different deep learning methods. (A) HU values difference between $\mathrm{CT}_{\mathrm{gt}}$ and

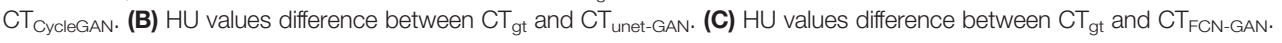

synthesized by $2 \mathrm{D}$ CycleGAN are the result of training after modifying the network layer of the $3 \mathrm{D}$ network and the loss function to the $2 \mathrm{D}$ mode. Figure $\mathbf{6 A}$ is the real CT images. Figures 6B, C show the $3 \mathrm{D}$ pseudo-CT images based on $3 \mathrm{D}$ CycleGAN and 2D CycleGAN with interpolation reconstruction. Figure 6D shows the CBCT images. Compared with the 2D results, the organ structures in the pseudo-CT images are more continuous in the $\mathrm{Z}$ direction. Table 3 shows the evaluation results between pseudo-CT and real CT images based on $3 \mathrm{D}$ and 2D CycleGAN under NMI and PSNR measurement methods, and the comparison results between CBCT and real CT images are used as a reference. The numerical results indicate that, compared with CBCT images, the HU values in pseudo-CT images obtained by the two CycleGAN methods are closer to the real CT images, but the $3 \mathrm{D}$ method is more accurate than the 2D method.

In addition, to prove the training effect of the gradient loss function on CycleGAN, we use CycleGAN with gradient loss and CycleGAN without it to perform pseudo-CT synthesis. The result is shown in Figure 7. For the CycleGAN method without gradient loss, the synthetic pseudo-CT image is generally fuzzy, caused by the L2 Euclidean distance loss function in the cyclic consistency loss function. In terms of details, the difference in areas with large gray gradient changes such as bones is more obvious, the edge information between organs is blurred, and the overall skin contour of patients is not accurate. Figures 7D, E also show that the improved CycleGAN with gradient loss can obtain pseudo CT with more accurate anatomical structure. In Figures 7D, E, the area with a bright visual effect is the $\mathrm{CT}_{\mathrm{gt}}$ with a window range of $(-600,600) \mathrm{HU}$, whereas the area with a dark visual effect is the pseudo CT with a window range of $(-400,800) \mathrm{HU}$.

\section{Dosimetric Evaluation}

In terms of dosimetry verification, the cross-sectional dose distributions of the treatment plan casting on $\mathrm{CT}_{\mathrm{gt}}$, $\mathrm{CT}_{\text {CycleGAN }}, \mathrm{CT}_{\text {unet-GAN }}, \mathrm{CT}_{\mathrm{FCN}-\mathrm{GAN}}$, and $\mathrm{CBCT}$ images for one of the testing patients were shown in Figures 8A-E. The experimental results showed that the dose distribution difference between $\mathrm{CT}_{\text {CycleGAN }}$ and $\mathrm{CT}_{\mathrm{gt}}$ in overall PTV was minor, and the dose distribution in the high-dose region of $\mathrm{CT}_{\text {CycleGAN }}$ was approximate to that of $\mathrm{CT}_{\mathrm{gt}}$. $\mathrm{PTV}$ is a region that includes part of the uterus, bladder, rectum, and other OARs. PTV is delineated with reference to the RTOG 63 report. The dose in the intersection region between the femoral head and PTV of $\mathrm{CT}_{\text {unet-GAN }}$ was deficient with inaccurate dose distribution. Many high-dose regions were found in PTV of $\mathrm{CT}_{\mathrm{FCN}-\mathrm{GAN}}$.

$\mathrm{CT}_{\mathrm{gt}}$ is compared with pseudo-CT images acquired through three methods in the DVH plot as shown in Figure 9, where the solid line is a DVH plot of a radiotherapy plan prepared based on patient $\mathrm{CT}_{\mathrm{gt}}$ image, and the dotted line is a DVH plot based on a pseudo-CT image. Figure 9A shows a DVH difference plot between $\mathrm{CT}_{\text {CycleGAN }}$ and $\mathrm{CT}_{\text {gt }}$. Figure 9B displays a $\mathrm{DVH}$ difference plot between $\mathrm{CT}_{\text {unet-GAN }}$ and $\mathrm{CT}_{\mathrm{gt}}$. Figure $\mathbf{9 C}$ is a $\mathrm{DVH}$ difference plot between $\mathrm{CT}_{\mathrm{FCN}-\mathrm{GAN}}$ and $\mathrm{CT}_{\mathrm{gt}}$. The overlapping degree of volumetric dose curves of multiple OARs in the DVH plot between $\mathrm{CT}_{\mathrm{CycleGAN}}$ and $\mathrm{CT}_{\mathrm{gt}}$ was the highest, and the volumetric dose curve difference in PTVs of the two was also small. In comparison with $\mathrm{CT}_{\text {CycleGAN, }}$ the volumetric dose curve of OARs in the DVH difference plot between $\mathrm{CT}_{\text {unet-GAN }}$ and $\mathrm{CT}_{\mathrm{gt}}$ was different, the volumetric dose curve of PTV and multiple OARs of $\mathrm{CT}_{\mathrm{FCN}-\mathrm{GAN}}$ differed considerably from that of $\mathrm{CT}_{\mathrm{gt}}$, and the volume of its highdose region was also large. Figure 9D shows the difference of $\mathrm{DVH}$ between CBCT and $\mathrm{CT}_{\mathrm{gt}}$. Table 4 lists the comparison results of dose indexes in PTV and OARs in the radiotherapy plan based on four CT images and one CBCT image. The average, maximum, and minimum doses in OARs and PTV in

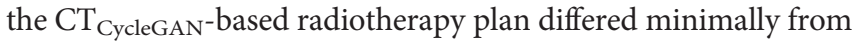
those in $\mathrm{CT}_{\mathrm{gt}}$-based radiotherapy plans. Figure $\mathbf{1 0}$ shows the comparison result of $\gamma$ analysis $(2 \% / 2 \mathrm{~mm})$ between the radiotherapy plan based on three types of pseudo-CT images and the radiotherapy plan based on $\mathrm{CT}_{\mathrm{gt}}$ images. We use $90 \% \gamma$ pass rate as the standard. The bluer the dots in the difference map, the smaller the dose difference between the two plans, and 
TABLE 2 | DSC (\%) measurement results of segmentation accuracy between real CT images and pseudo-CT images synthesized by three different methods.

\begin{tabular}{|c|c|c|c|}
\hline \multirow[t]{2}{*}{ Organ } & \multicolumn{3}{|c|}{ Method } \\
\hline & CycleGAN & Unet-GAN & FCN-GAN \\
\hline bladder & $91.58 \pm 0.45^{\star}$ & $88.63 \pm 0.51$ & $87.83 \pm 0.56$ \\
\hline uterus & $88.14 \pm 1.26^{\star}$ & $87.52 \pm 1.60$ & $85.31 \pm 2.72$ \\
\hline rectum & $87.23 \pm 2.01^{*}$ & $85.64 \pm 2.33$ & $84.39 \pm 3.51$ \\
\hline bone & $92.59 \pm 0.33^{\star}$ & $89.57 \pm 0.47$ & $86.78 \pm 0.58$ \\
\hline
\end{tabular}

*Indicates significant improvement via paired $t$-tests $(p<0.05)$.

The bold is used to show the best performance.

A
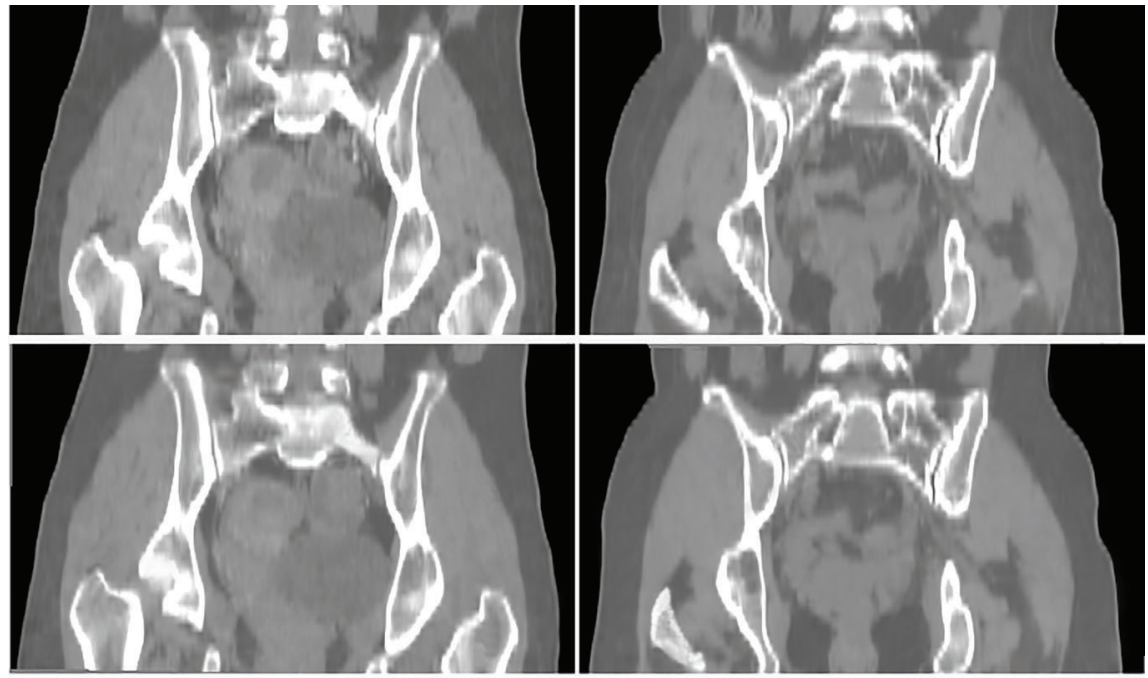

C
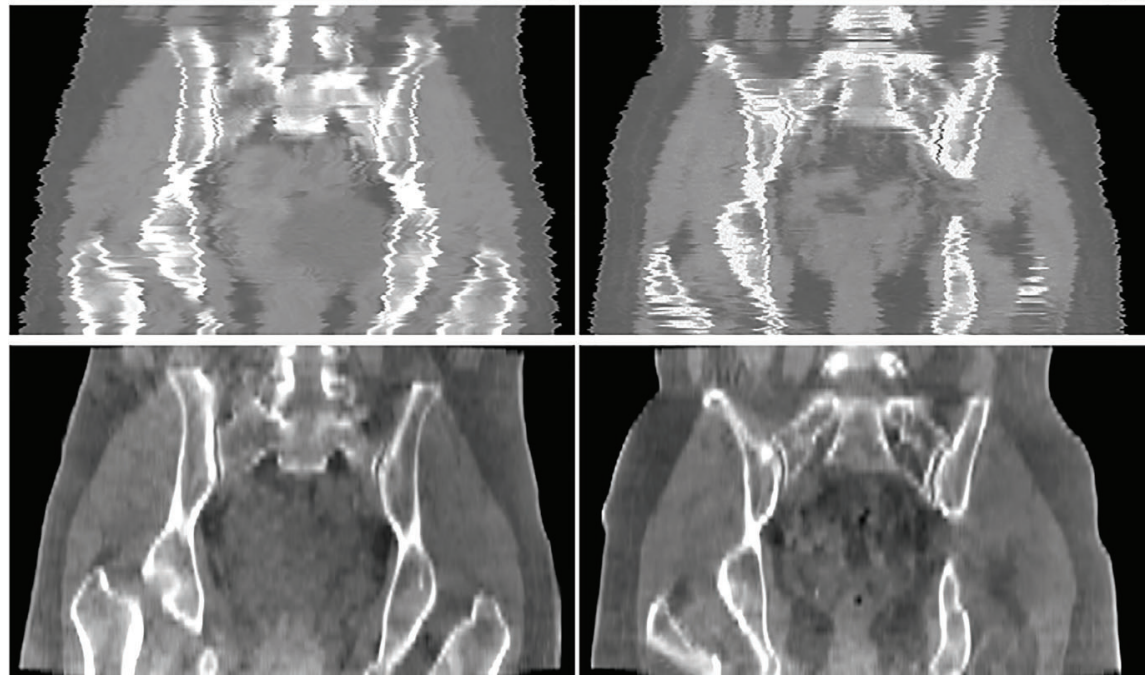

FIGURE 6 | Comparison results of $\mathrm{CT}_{\mathrm{gt}}$ and pseudo CT obtained based on 3D and 2D CycleGAN. (A) real CT images(CT $\mathrm{gt}_{\mathrm{t}}$ ) (B) pseudo-CT images obtained based on 3D CycleGAN. (C) pseudo-CT images obtained based on 2D CycleGAN. (D) CBCT images.

the higher the overall $\gamma$-pass rate. The three plans based on $\mathrm{CT}_{\text {CycleGAN }}, \mathrm{CT}_{\text {unet-GAN }}$, and $\mathrm{CT}_{\mathrm{FCN}-\mathrm{GAN}}$ had a $\gamma$-pass rate of $97.0 \%, 93.7 \%$, and $84.9 \%$ respectively, indicating that the dose difference between the plans based on $\mathrm{CT}_{\text {CycleGAN }}$ and $\mathrm{CT}_{\mathrm{gt}}$ was the smallest.

\section{DISCUSSION}

Pseudo-CT images acquired through deep learning methods based on CBCT images have various advantages in clinical radiotherapy and can solve the poor $\mathrm{CBCT}$ imaging quality of 
TABLE 3 | Metric results between real CT images and different CT images of 20 patients with cervical cancer.

\begin{tabular}{|c|c|c|c|}
\hline \multirow[t]{2}{*}{ Metric } & \multicolumn{3}{|c|}{ CT images } \\
\hline & $\mathrm{CT}_{\text {CycleGAN-3D }}$ & $\mathrm{CT}_{\text {CycleGAN-2D }}$ & CBCT \\
\hline $\mathrm{NMI}$ & $0.90 \pm 0.01$ & $0.87 \pm 0.02$ & $0.79 \pm 0.02$ \\
\hline PSNR (dB) & $30.70 \pm 0.78$ & $29.72 \pm 0.59$ & $27.15 \pm 0.57$ \\
\hline
\end{tabular}

CT CycleGAN-3D, pseudo-CT images obtained based on 3D CycleGAN; CT CycleGAN-2D, pseudo-CT images obtained based on 2D CycleGAN.
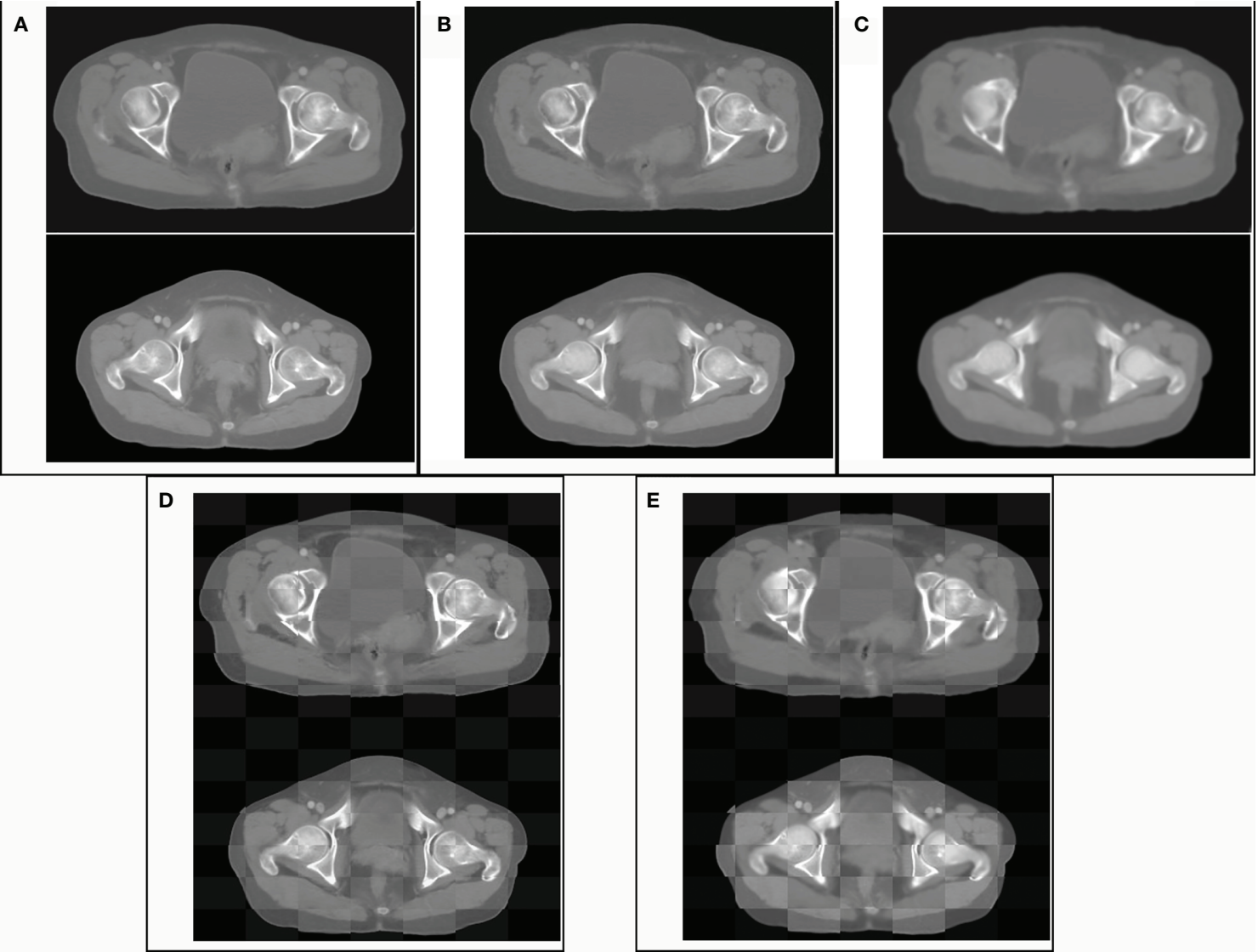

FIGURE 7 | Results of pseudo-CT images obtained by adding gradient loss function and not adding it in CycleGAN. (A) CT gt. (B) pseudo CT obtained by CycleGAN with gradient loss. (C) pseudo CT obtained based on CycleGAN without gradient loss. (D) The comparison result of (A, B). (E) Comparison result of (A, C).

soft tissues and the impossible direct correction of an adaptive radiotherapy plan. In clinical $\mathrm{ART}$, a $\mathrm{CT}_{\mathrm{gt}}$ image applied to plan correction is acquired based on an image registration algorithm. This method needs to set the corresponding objective function according to the complexity of the registration region or anatomical structure. The non-rigid registration method is timeconsuming for image registration tasks with complex texture information. Given that the boundary between the soft tissues of the $\mathrm{CT}(\mathrm{CBCT})$ image is not clear, the registration accuracy is not accurate. The CycleGAN-based deep learning method can construct the nonlinear mapping relationship between two image domains by a multilayer convolutional neural network with high feature extraction effect and efficiency, so it can solve the disadvantages of the deformation registration method.

According to comparative experimental results in the aspects of anatomical structure and dosimetry, the FCN-GAN-based method has unsatisfactory results in the skeleton region of synthetic rigid structure and the soft tissue region of nonrigid structure because paired data after registration are needed in FCN-GAN training. Otherwise, data mismatching and distortion can be easily caused. Moreover, the generator based on a fully convolutional neural network only contains convolutional layers without a shortcut connection of residual network, so it fails to combine superficial-layer features with deep-layer features. 

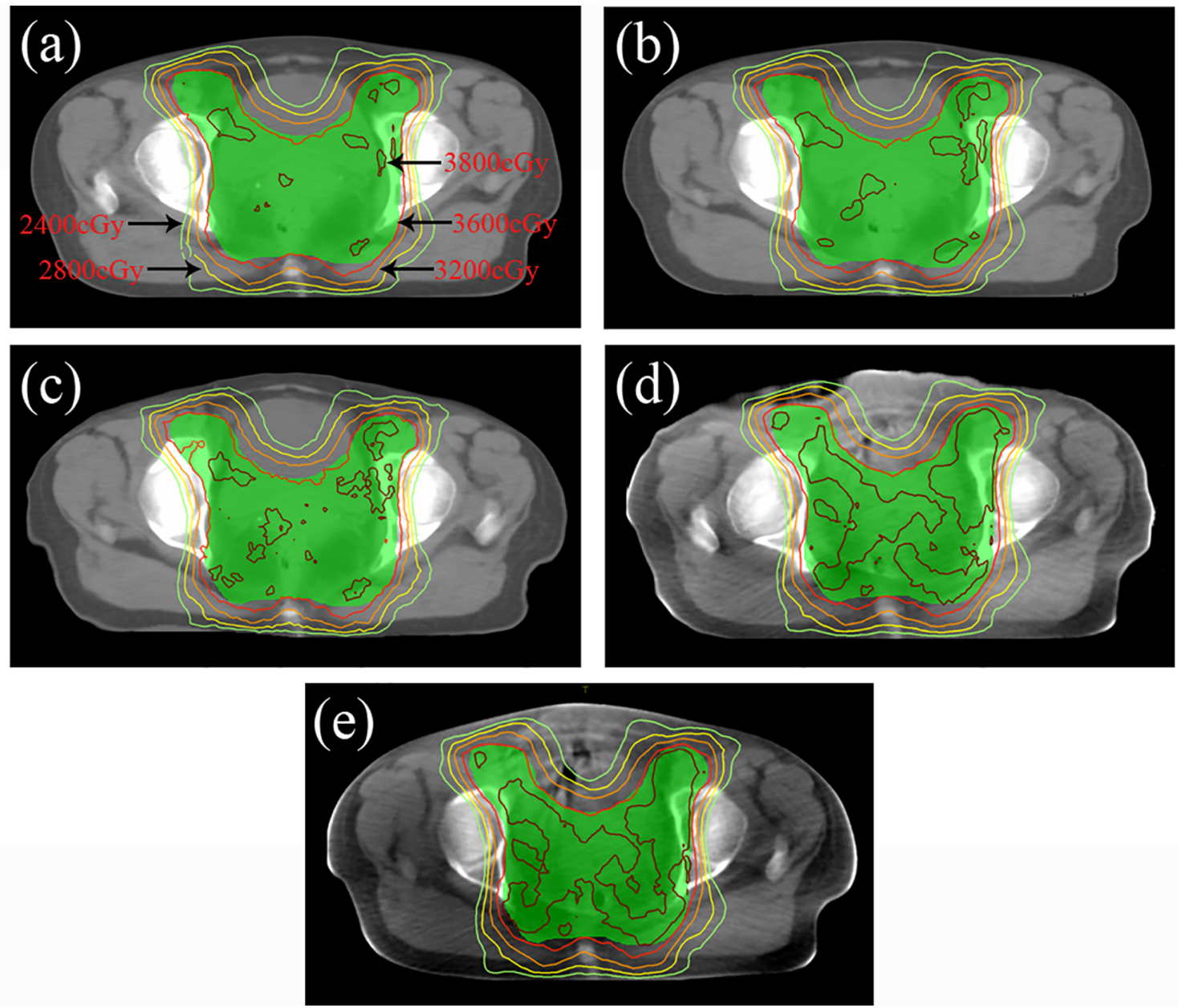

FIGURE 8 | Dose distribution of radiotherapy plan based on four CT images at the isocenter level. (A) Dose distribution of CT $\mathrm{CT}_{\text {CycleGAN }}$ at isocenter. (C) Dose distribution of $\mathrm{CT}_{\text {unet-GAN }}$ at isocenter. (D) Dose distribution of $\mathrm{CT}_{\mathrm{FCN}}$-GAN at isocenter. (E) Dose distribution of CBCT at isocenter.

Consequently, the accuracy of model synthesis is degraded. The Unet-GAN-based method has a better synthetic effect than the former, but partial deformation still takes place in the skeleton region and some soft tissues are also inaccurate because preprocessed paired volume data are also needed for its training. A pooling layer is included in the U-Net network, resulting in losses of feature information of some anatomical structures. Then, the HU value in the synthesized pseudo-CT image is inaccurate. The improved CycleGAN network method proposed in this study can acquire pseudo-CT images better. Compared with a conventional GAN network only containing an adversarial loss function, the CycleGAN carrying cycle loss is a GAN synthetic network that contains two symmetrical mapping relations $(\mathrm{CBCT} \rightarrow \mathrm{CT}$ and $\mathrm{CT} \rightarrow \mathrm{CBCT})$. The cycle loss function is L2 Euclidean distance between the input of the original image domain and pseudo image output in the same image domain acquired by twice feature transformation. Based on this bidirectional feature transformation pattern, the model can be trained without needing paired data (34). Owing to the existence of deformation and positioning set up errors of soft tissues to different degrees, the image data of the same patient acquired in different periods during the clinical radiotherapy are not the same, but the CycleGAN network can train unpaired CBCT-CT volume data to acquire pseudo image data, so it conforms to clinical practical application. However, $3 \mathrm{D}$ volume data are rich in feature information, so guaranteeing the accuracy of texture feature information of synthesized images only through the traditional loss function. Thus, the $3 \mathrm{D}$ gradient loss function was added into the objective function in this study to reserve detailed information of pseudo-CT images as much as possible.

An encoding-decoding pattern with a residual connection is used for the generator of the 3D CycleGAN network. Under this pattern, the output sharing the same dimensionality with the input is acquired through down-sampling, feature transformation, and up-sampling operations of the input volume data. Redundant feature information in the image can be compressed, to effectively extract image feature information (35). This network will splice the output of the previous layer 


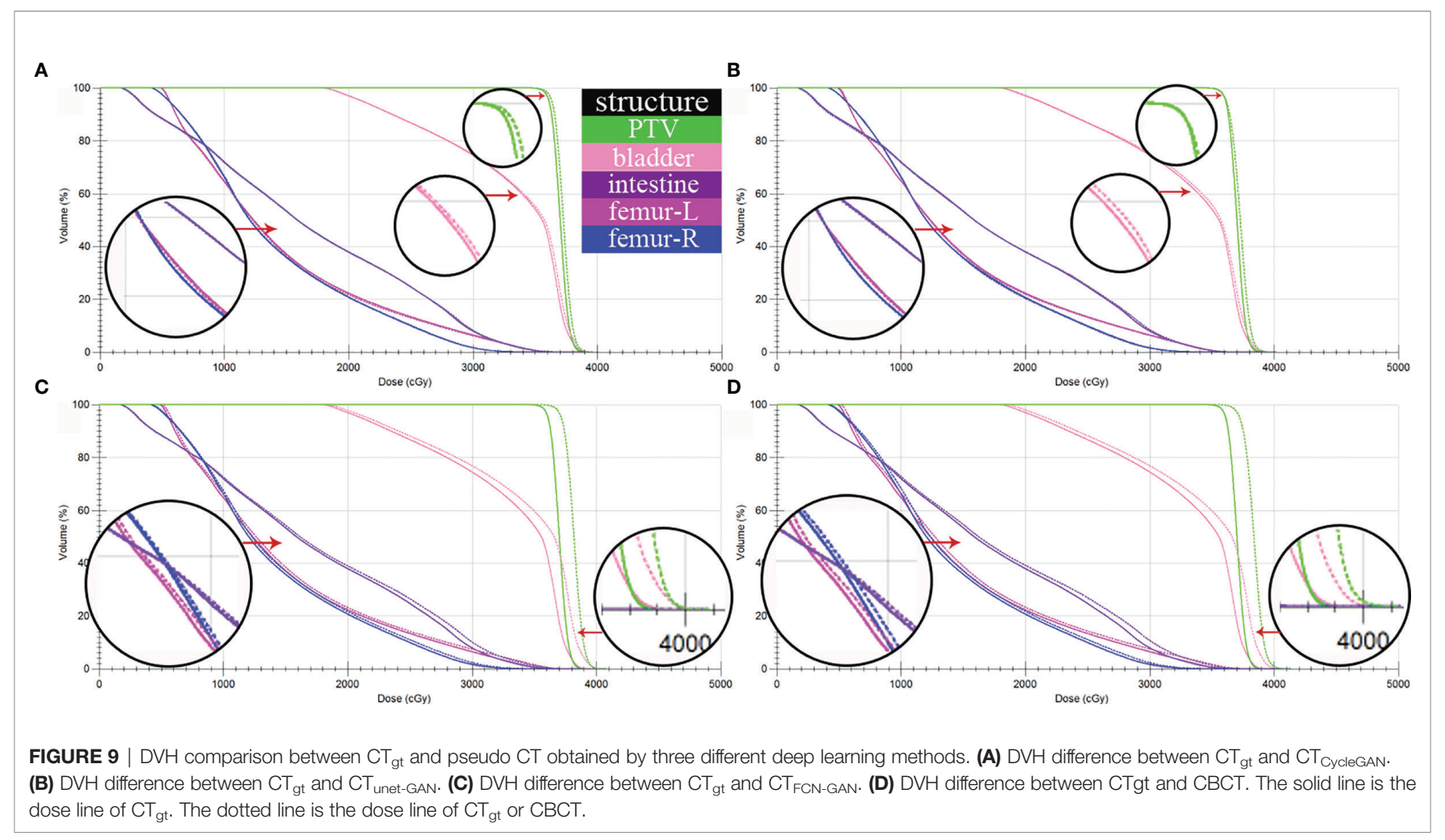

TABLE 4 | Comparison of dose indexes of PTV and OARs in four CT images based on Monte Carlo optimization.

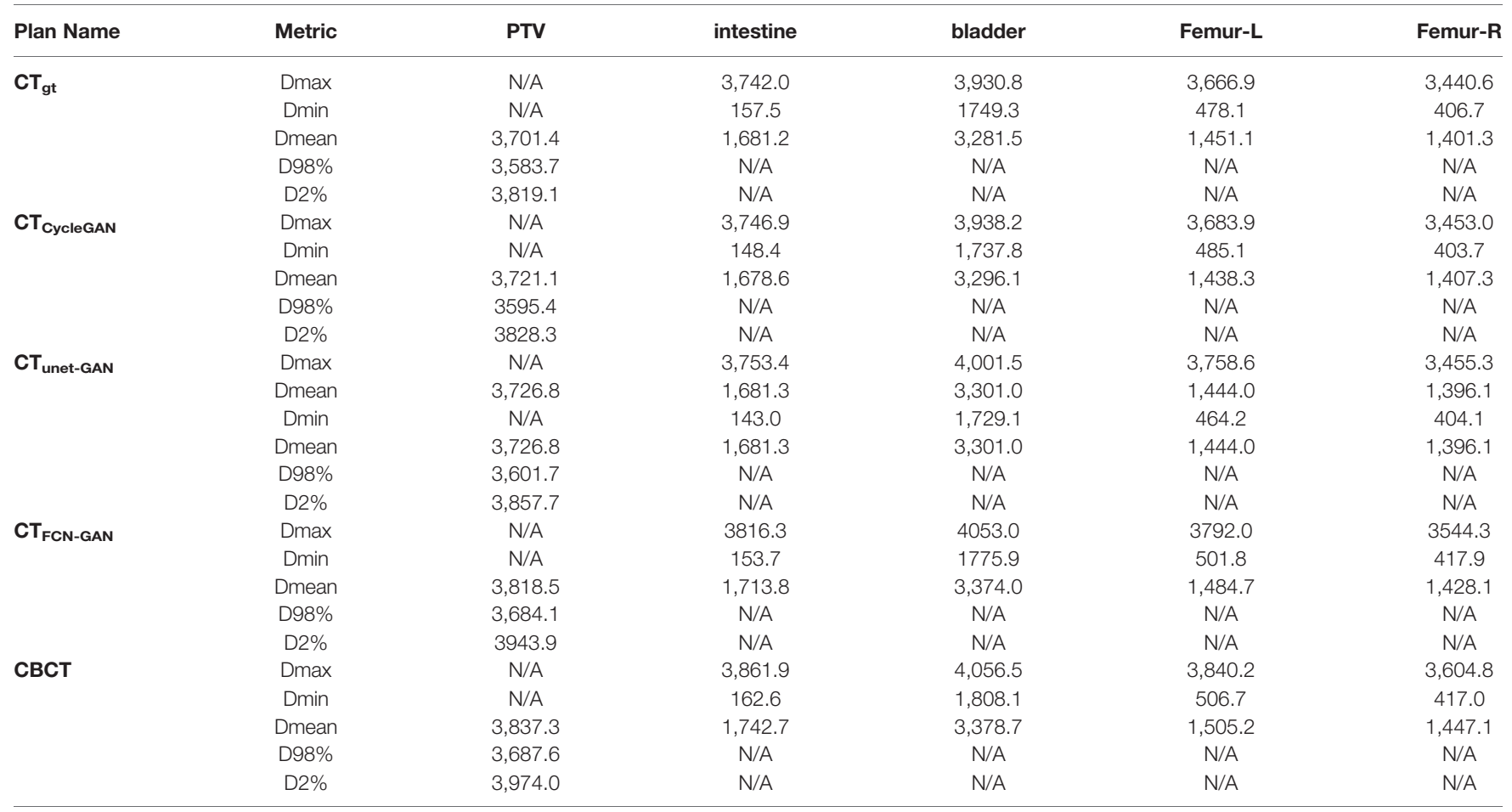

Dmax, maximum dose; Dmean, mean dose; Dmin, minimum dose; D98\%, dose covering 98\% of the PTV; D2\%, dose to $2 \%$ of the PTV. 

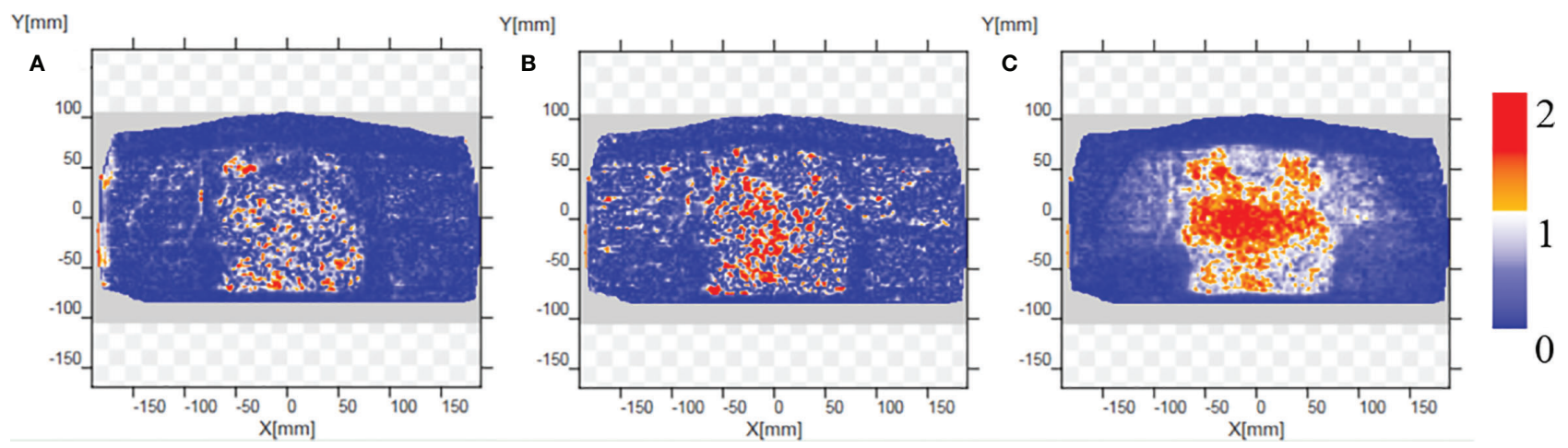

FIGURE 10 | Comparison results of $\gamma$ analysis $(2 \% / 2 \mathrm{~mm})$ between the radiotherapy plan based on three types of pseudo-CT images and the radiotherapy plan based on $\mathrm{CT}_{\text {gt }}$ images. The bluer the dots in the difference map, the smaller the dose difference between the two plans, and the higher the overall $\gamma$-pass rate.

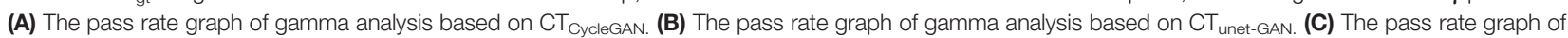

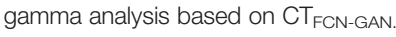

with the output of the most adjacent convolution block before each up-sampling layer. This residual connection mode ensures integrating features of different layers during pseudo-CT synthesis. When increasing the network depth, this mode can improve the utilization efficiency of volume data features, acquiring accurate pseudo-CT images. In addition, if the network is operated by using $3 \mathrm{D}$ volume data of global dimensions as the input, considerable GPU video memory will be consumed, so parallel input mode based on image block can help to extract additional local feature information of the image while saving training time and reducing video memory consumption (36).

All data adopted in this study were volume data of abdominal cervical cancer patients. Relative to head and neck images, abdominal images acquired in different periods could have evident deformation changes in their internal soft tissues and OARs. The accuracy and reliability of images generated based on the CycleGAN could be better verified. Furthermore, the $3 \mathrm{D}$ pseudo-CT image acquired based on a 3D training model had spatial information in the $\mathrm{Z}$ direction, and it could not only be applied to positioning verification but also to the correction of radiotherapy plans. The pseudo-CT images synthesized by $2 \mathrm{D}$ CycleGAN cannot guarantee the continuity of the $\mathrm{Z}$ direction. Each layer is synthesized independently, leading to false registration between the images in two image domains in the 2D image synthesis task, resulting in false pseudo-CT synthesis. For example, establishing a mapping relationship between one CBCT slice and multiple CT slices is possible. If the pseudo-CT images synthesized based on 2D CycleGAN are not subjected to image post-processing, the final reconstructed $3 \mathrm{D}$ images cannot easily be used in clinical radiotherapy.

The pseudo-CT acquisition method based on 3D CycleGAN also has limitations. Given that the network contains GAN networks in two synthetic directions, the training speed is lower than that of unidirectional GAN. A pseudo-CT image with a satisfactory effect can be acquired only by multiple epochs. In the subsequent experiment, patch image blocks of different input dimensions will be adjusted by debugging hyperparameters of generators and discriminators, like learning rate, to elevate the training speed. In addition, multiple regions of interest will be divided according to density differences of OARs and different objective functions will be set to realize stepwise pseudo-CT synthesis, thereby further improving imaging quality of pseudoCT images in the aspect of local details.

\section{CONCLUSION}

An improved method of acquiring pseudo-CT images based on a 3D CycleGAN network with residual connections and attention gates was raised in this study. In the aspect of anatomical structure verification, the similarity degree of texture greyscale information of pseudo-CT images obtained through the new method with that of $\mathrm{CT}_{\mathrm{gt}}$ images was experimentally proven higher in comparison with other GAN deep learning methods. For the sake of dosimetry verification, the dose distributions between radiotherapy plans prepared based on $\mathrm{CT}_{\mathrm{gt}}$ image and those prepared based on pseudo-CT images acquired through the improved method were approximate under the same optimization conditions. Owing to its capability of eliminating the disadvantages of CBCT images in practical clinical application, the pseudo-CT image has outstanding application prospects in adaptive radiotherapy of cervical cancer.

\section{DATA AVAILABILITY STATEMENT}

The raw data supporting the conclusions of this article will be made available by the authors, without undue reservation.

\section{ETHICS STATEMENT}

The studies involving human participants were reviewed and approved by the medical ethics committee of the Second People's 
Hospital of Changzhou, Nanjing Medical University (2017-00201 ). The patients/participants provided their written informed consent to participate in this study.

\section{AUTHOR CONTRIBUTIONS}

HS performed the experimental characterizations and analyzed the results. RF focused on the image segmentation and assisted to train the 3D CycleGAN. CL assisted to perform the experimental characterizations and worked out a radiotherapy plan. ZL collected the image data assisted to preprocess the images. $\mathrm{KX}$ assisted to collected the image data and evaluated the

\section{REFERENCES}

1. Eichenauer DA, Aleman BMP, André M, Federico M, Hutchings M, Illidge T, et al. Hodgkin lymphoma: ESMO Clinical Practice Guidelines for diagnosis, treatment and follow-up. Ann Oncol (2018) 29:19-29. doi: 10.1093/annonc/ mdy080

2. Toita T, Ariga T, Kasuya G, Hashimoto S, Maemoto H, Heianna J, et al. Current Status and Perspective of Chemoradiotherapy for Uterine Cervical Cancer. Gan to kagaku ryoho. Cancer Chemother (2015) 42(10):1156-61.

3. Rodriguez N, Algara M, Foro P, Lacruz M, Reig A, Membrive I, et al. Predictors of Acute Esophagitis in Lung Cancer Patients Treated With Concurrent Three-Dimensional Conformal Radiotherapy and Chemotherapy. Int J Radiat Oncol Biol Phys (2009) 73(3):810-7. doi: 10.1016/j.ijrobp.2008.04.064

4. Quint S, Hoogeman MS, Ahmad R, Dhawtal G, Bondar L, De Pree I, et al. IGRT in EBRT for cervical cancer, a plan of the day strategy. Radiother Oncol (2009) 92:S100-1. doi: 10.1016/S0167-8140(12)72856-6

5. Liu C, Kumarasiri A, Chetty IJ, Kim J. SU-E-J-206: Delivered Dose to Organs From CBCT-Based IGRT of the Prostate. Med Phys (2013) 40(6):199-200. doi: 10.1118/1.4814418

6. Meroni S, Mongioj V, Giandini T, Bonfantini F, Cavallo A, Carrara M, et al. EP-1822: limits and potentialities of the use of CBCT for dose calculation in adaptive radiotherapy. Radiother Oncol (2016) 119:S854-5. doi: 10.1016/ S0167-8140(16)33073-0

7. Siewerdsen JH, Moseley DJ, Bakhtiar B, Richard S, Jaffray DA. The influence of antiscatter grids on soft-tissue detectability in cone-beam computed tomography with flat-panel detectors. Med Phys (2004) 31(12):3506-20. doi: 10.1118/1.1819789

8. Mail N, Moseley DJ, Siewerdsen JH, Jaffray DA. The influence of bowtie filtration on cone-beam CT image quality. Med Phys (2009) 36(1):22-32. doi: $10.1118 / 1.3017470$

9. Kyriakou Y, Riedel T, Kalender WA. Combining deterministic and Monte Carlo calculations for fast estimation of scatter intensities in CT. Phys Med Biol (2006) 51(18):4567-86. doi: 10.1088/0031-9155/51/18/008

10. Yang X, Wu S, Sechopoulos I, Fei B. Cupping artifact correction and automated classification for high-resolution dedicated breast CT images. Med Phys (2012) 39(10):6397-406. doi: 10.1118/1.4754654

11. Qu X, Lai CJ, Zhong Y, Yi Y, Shaw CC. A general method for cupping artifact correction of cone-beam breast computed tomography images. Comput Assisted Radiol Surg (2016) 11(7):1233-46. doi: 10.1007/s11548-015-1317-8

12. Usui K, Inoue T, Kurokawa C, Sugimoto S, Ogawa K. SU-F-J-70: Monte Carlo Study On a Cone-Beam Computed Tomography Using a Cross-Type Carbon Fiber Antiscatter Grid. Med Phys (2016) 43(6):3422-5. doi: 10.1118/1.4955978

13. Chevillard C, Dumas JL, Mazal A, Husson F. 42. Computation of the RT dose of the day from mapping CBCT information to the planning CT using an optimized elastic registration method. Physica Med (2017) 44:20-1. doi: 10.1016/j.ejmp.2017.10.067

14. Derksen A, Koenig L, Meine H, Heldmann S. SU-F-J-97: A Joint Registration and Segmentation Approach for Large Bladder Deformations radiotherapy plan. XN conceived the experiments and revised the manuscript. JY revised the manuscript. All authors contributed to the article and approved the submitted version.

\section{FUNDING}

This work was supported in part by the General Program of Jiangsu Provincial Health Commission (No. M2020006), in part by the Changzhou Key Laboratory of Medical Physics (No. CM20193005), and in part by the Innovation Foundation for Doctor Dissertation of Northwestern Polytechnical University (No. CX202039).

in Adaptive Radiotherapy. Med Phys (2016) 43(6):3429-9. doi: 10.1118/ 1.4956005

15. Abe T, Tateoka K, Saito Y, Nakazawa T, Yano M, Nakata K, et al. Method for Converting Cone-Beam CT Values into Hounsfield Units for Radiation Treatment Planning. Int J Med Phys Clin Eng Radiat Oncol (2017) 06 (4):361-75. doi: 10.4236/ijmpcero.2017.64032

16. Lei Y, Tang X, Higgins KA, Lin J, Jeong J, Liu T, et al. Learning-based CBCT correction using alternating random forest based on auto-context model. Med Phys (2018) 46(2):601-18. doi: 10.1002/mp.13295

17. Wang H, Barbee D, Wang W, Pennell R, Osterman K. SU-F-J-109: Generate Synthetic CT From Cone Beam CT for CBCT-Based Dose Calculation. Med Phys (2016) 43(6Part10):3432-2. doi: 10.1118/1.4956017

18. Zhu JY, Park T, Isola P, Efros AA. Unpaired image-to-image translation using cycle-consistent adversarial networks. In: Proceedings of the IEEE international conference on computer vision. Venice: IEEE (2017). p. 2223-32. doi: 10.1109/ ICCV.2017.244

19. Liang X, Chen L, Nguyen D, Zhou Z, Gu X, Yang M, et al. Generating synthesized computed tomography (CT) from cone-beam computed tomography (CBCT) using CycleGAN for adaptive radiation therapy. Phys Med Biol (2019) 64(12):125002. doi: 10.1088/1361-6560/ab22f9

20. Kida S, Kaji S, Nawa K, Imae T, Nakamoto T, Ozaki S, et al. Visual enhancement of Cone-beam CT by use of CycleGAN. Med Phys (2020) 47 (3):998-1010. doi: 10.1002/mp.13963

21. Chen H, Dou Q, Yu L, Qin J, Heng PA. VoxResNet: Deep voxelwise residual networks for brain segmentation from 3D MR images. Neuroimage (2017) 170:S1053811917303348. doi: 10.1016/j.neuroimage.2017.04.041

22. Qiao Z, Cui Z, Niu X, Geng S, Yu Q, et al. Image Segmentation with Pyramid Dilated Convolution Based on ResNet and U-Net. In: International Conference on Neural Information Processing. Cham: Springer (2017).

23. Kudo Y, Aoki Y. Dilated convolutions for image classification and object localization. In: 2017 Fifteenth IAPR International Conference on Machine Vision Applications (MVA). Nagoya: IEEE (2017). p. 452-5. doi: 10.23919/ MVA.2017.7986898

24. He K, Zhang X, Ren S, Jian S. Deep residual learning for image recognition. Comput Vision Pattern Recog (2016) 770-8. doi: 10.1109/CVPR.2016.90

25. Liu Y, Lei Y, Wang T, Fu Y, Tang X, Curran WJ, et al. CBCT-based Synthetic CT Generation using Deep-attention CycleGAN for Pancreatic Adaptive Radiotherapy. Med Phys (2020) 47(6):2472-83. doi: 10.1002/mp.14121

26. Zhao J, Zhang J, Li Z, Hwang JN, Gao Y, Fang Z, et al. DD-CycleGAN: Unpaired image dehazing via Double-Discriminator Cycle-Consistent Generative Adversarial Network. Eng Appl Artif Intell (2019) 82(JUN.):26371. doi: 10.1016/j.engappai.2019.04.003

27. Zhou Z, Wang Y, Guo Y, Qi Y, Yu J. Image Quality Improvement of HandHeld Ultrasound Devices With a Two-Stage Generative Adversarial Network. IEEE Trans Biomed Eng (2020) 67(1):298-311. doi: 10.1109/TBME.2019.2912986

28. Nie D, Trullo R, Lian J, Petitjean C, Ruan S, Wang Q, et al. Medical Image Synthesis with Context-Aware Generative Adversarial Networks. In: Medical image computing and computer assisted intervention. Cham: Springer (2017). p. 417-25. doi: 10.1007/978-3-319-66179-7_48 
29. Lee J, Nishikawa R, Reiser I, Boone J. WE-G-207-05: Relationship Between CT Image Quality, Segmentation Performance, and Quantitative Image Feature Analysis. Med Phys (2015) 42(6):3697-7. doi: 10.1118/1.4926098

30. He K, Cao X, Shi Y, Nie D, Gao Y, Shen D. Pelvic Organ Segmentation Using Distinctive Curve Guided Fully Convolutional Networks. IEEE Trans Med Imaging (2019) 38(2):585-95. doi: 10.1109/TMI.2018.2867837

31. Nithiananthan S, Schafer S, Mirota DJ, Stayman JW, Zbijewski W, Reh DD, et al. Extra-dimensional Demons: A method for incorporating missing tissue in deformable image registration. Med Phys (2012) 39(9):5718-31. doi: $10.1118 / 1.4747270$

32. Hore A, Ziou D. Image quality metrics: PSNR vs. SSIM//2010 20th International Conference on Pattern Recognition. IEEE (2010) 2366-9. doi: 10.1109/ICPR.2010.579

33. Wendling M, Zijp LJ, Mcdermott LN, Smit EJ, Sonke JJ, Mijnheer BJ, et al. A fast algorithm for gamma evaluation in 3D. Med Pehysics (2007) 34(5):164754. doi: $10.1118 / 1.2721657$

34. Almahairi A, Rajeswar S, Sordoni A, Bachman P, Courville A. Augmented CycleGAN: Learning Many-to-Many Mappings from Unpaired Data. Int Conf Mach Learn (2018) 195-204.
35. Chen H, Zhang Y, Kalra MK, Lin F, Chen Y, Liao P, et al. Low-Dose CT With a Residual Encoder-Decoder Convolutional Neural Network. IEEE Trans Med Imaging (2017) 36(12):2524-35. doi: 10.1109/TMI. 2017.2715284

36. Long J, Shelhamer E, Darrell T. Fully Convolutional Networks for Semantic Segmentation. IEEE Trans Pattern Anal Mach Intell (2015) 39(4):640-51. doi: 10.1109/TPAMI.2016.2572683

Conflict of Interest: The authors declare that the research was conducted in the absence of any commercial or financial relationships that could be construed as a potential conflict of interest.

Copyright (c) 2021 Sun, Fan, Li, Lu, Xie, Ni and Yang. This is an open-access article distributed under the terms of the Creative Commons Attribution License (CC BY). The use, distribution or reproduction in other forums is permitted, provided the original author(s) and the copyright owner(s) are credited and that the original publication in this journal is cited, in accordance with accepted academic practice. No use, distribution or reproduction is permitted which does not comply with these terms. 